\title{
A LOFAR study of non-merging massive galaxy clusters
}

\author{
F. Savini ${ }^{1}$, A. Bonafede ${ }^{1,2,3}$, M. Brüggen ${ }^{1}$, D. Rafferty ${ }^{1}$, T. Shimwell ${ }^{4,5}$, A. Botteon $^{2,3}$, G. Brunetti $^{2}$, H. Intema ${ }^{4}$, \\ A. Wilber ${ }^{1}$, R. Cassano ${ }^{2}$, F. Vazza ${ }^{3}$, R. van Weeren ${ }^{4}$, V. Cuciti ${ }^{2,3}$, F. De Gasperin ${ }^{1}$, H. Röttgering ${ }^{4}$, M. Sommer ${ }^{6}$, \\ L. Bîrzan ${ }^{1}$, and A. Drabent ${ }^{7}$
}

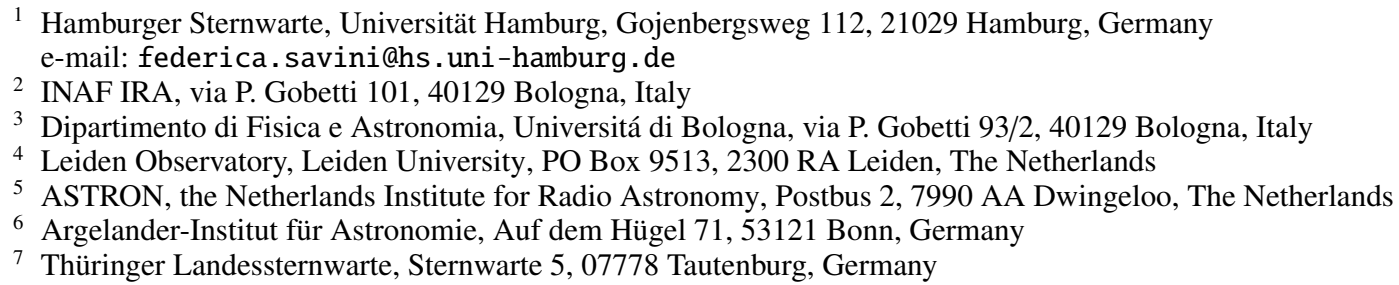

Received 16 July 2018 / Accepted 11 September 2018

\begin{abstract}
Centrally located diffuse radio emission has been observed in both merging and non-merging galaxy clusters. Depending on their morphology and size, we distinguish between giant radio haloes, which occur predominantly in merging clusters, and mini haloes, which are found in non-merging, cool-core clusters. In recent years, cluster-scale radio emission has also been observed in clusters with no sign of major mergers, showing that our knowledge of the mechanisms that lead to particle acceleration in the intra-cluster medium (ICM) is still incomplete. Low-frequency sensitive observations are required to assess whether the emission discovered in these few cases is common in galaxy clusters or not. With this aim, we carried out a campaign of observations with the LOw Frequency ARay (LOFAR) in the frequency range $120-168 \mathrm{MHz}$ of nine massive clusters selected from the Planck SZ catalogue, which had no sign of major mergers. In this paper, we discuss the results of the observations that have led to the largest cluster sample studied within the LOFAR Two-metre Sky Survey, and we present Chandra X-ray data used to investigate the dynamical state of the clusters, verifying that the clusters are currently not undergoing major mergers, and to search for traces of minor or off-axis mergers. We discover large-scale steep-spectrum emission around mini haloes in the cool-core clusters PSZ1G139.61+24 and RXJ1720.1+2638, which is not observed around the mini halo in the non-cool-core cluster A1413. We also discover a new $570 \mathrm{kpc}-\mathrm{halo}$ in the noncool-core cluster RXCJ0142.0+2131. We derived new upper limits to the radio power for clusters in which no diffuse radio emission was found, and we discuss the implication of our results to constrain the cosmic-ray energy budget in the ICM. We conclude that radio emission in non-merging massive clusters is not common at the sensitivity level reached by our observations and that no clear connection with the cluster dynamical state is observed. Our results might indicate that the sloshing of a dense cool core could trigger particle acceleration on larger scales and generate steep-spectrum radio emission.
\end{abstract}

Key words. techniques: interferometric - acceleration of particles - surveys - galaxies: clusters: general galaxies: clusters: intracluster medium - radio continuum: general

\section{Introduction}

Diffuse radio emission in galaxy clusters is caused by relativistic electrons that emit synchrotron radiation in the intra-cluster magnetic field (see Feretti et al. 2012 and references therein). This emission has a low surface brightness $\left(\sim 0.1-1 \mu \mathrm{Jy} \operatorname{arcsec}^{-2}\right.$ at $v \sim 1.4 \mathrm{GHz})$ and, depending on its morphology, location, and size (hundreds of kpc up to few $\mathrm{Mpc}$ ), it is classified as radio relic, radio halo, or mini halo. In this paper, we are only concerned with radio haloes and mini haloes, which are sources that are located at the centres of galaxy clusters. Both type of sources are commonly characterised by a steep radio spectrum with a spectral index of ${ }^{1} \alpha<-1$.

Giant radio haloes ( $\sim \mathrm{Mpc}$-scale) have predominantly been found in merging clusters (e.g. Buote 2001, Cassano et al. 2010, Cuciti et al. 2015) that typically do not have a cool core, i.e. a core characterised by a peaked X-ray surface brightness, high

\footnotetext{
1 The spectrum is defined by $S(v) \propto v^{\alpha}$.
}

gas density, and significant drop in temperature within the inner $\sim 100 \mathrm{kpc}$. Haloes indicate the presence of a cluster-wide particle acceleration mechanism, such as in situ re-acceleration (see Brunetti \& Jones 2014 for a review).

Mini haloes $(<500 \mathrm{kpc}$-scale) are detected in cool-core clusters that have not been disrupted or disturbed by major mergers. Often, the brightest cluster galaxy (BCG) resides at the centre of the mini halo and, when the BCG is radio loud, its radio lobes are embedded in the mini halo. The origin of the relativistic cosmic-ray electrons $(\mathrm{CRe})$ of mini haloes is still unclear. The main contending theories are either leptonic (re-acceleration) models and hadronic models. Leptonic models involve the re-acceleration of a lower energy population of CRe by turbulent motions in the intra-cluster medium (ICM; Gitti et al. 2002). The turbulence can be caused by the sloshing of the low-entropy gas falling inside the dark matter potential well of the cluster. The sloshing can be instigated, for example, by a minor merger with a lower mass galaxy cluster or group. 
When the gas meets the higher entropy ICM, a cold front, i.e. a discontinuity in the X-ray emissivity, is formed (ZuHone et al. 2013). Hadronic models involve the injection of secondary electrons produced continuously by inelastic collisions of relativistic cosmic-ray protons (CRp) with the cluster thermal proton population (Pfrommer \& Enßlin 2004). The CRp are expected to be present in the ICM, therefore some level of synchrotron emission from secondary electrons is expected in galaxy clusters, especially in non-merging clusters with a dense cool core, where the rate of hadronic interactions should be the highest.

Recent observations with the Giant Metrewave Radio Telescope (GMRT) and the Very Large Array (VLA) have revealed the presence of radio haloes in a few clusters that are not undergoing major mergers, and that - in some cases - host a cool core (i.e. CL1821+643, Bonafede et al. 2014; A2261, A2390, Sommer et al. 2017; A2142, Venturi et al. 2017), challenging the notion that radio haloes only form in major mergers. It has been proposed that these sources might be connected to the occurrence of minor/off-axis mergers, although it remains unclear how minor mergers could initiate continuum emission on megaparsec scales. Models (e.g. Cassano et al. 2006) predict that minor mergers can generate ultra-steep spectrum (USS) emission $(\alpha<$ $-1.5)$ due to the smaller amount of energy available compared to major mergers. However, flatter spectrum sources such as that in CL1821+643, where $\alpha_{323}^{1665} \sim-1.1$, are difficult to explain in this scenario. Finally, we note that signatures of minor-merging activities and gas-sloshing mechanisms have been detected in clusters containing mini haloes (e.g. Gitti et al. 2007; Giacintucci et al. 2014a, Savini et al. 2018a). To assess whether the emission discovered in these few cases is common in galaxy clusters or not if looking at low radio frequencies, we selected and studied a sample of nine non-merging galaxy clusters. The observations were carried out with the LOw Frequency ARray (LOFAR; van Haarlem et al. 2013) with the aim of studying large-scale radio emission to shed light on the non-thermal phenomena in galaxy clusters and the connection with cluster dynamics. Sensitive low-frequency observations allow us to detect steep-spectrum emission that cannot be observed at higher frequencies and are fundamental in the case of slightly disturbed clusters.

In case of the non-detection of large-scale diffuse sources, we provide new upper limits on the radio power of haloes. These constraints demonstrate the importance of low-frequency observations to determine the cosmic-ray pressure and energy budget in clusters.

In this paper, we assume a flat, $\Lambda$ cold dark matter cosmology with matter density $\Omega_{\mathrm{M}}=0.3$ and Hubble constant $H_{0}=67.8 \mathrm{~km} \mathrm{~s}^{-1} \mathrm{Mpc}^{-1}$ (Planck Collaboration XIII 2016). All our images are in the $\mathrm{J} 2000$ coordinate system.

\section{The sample}

In order to investigate the presence of diffuse emission in nonmerging systems at low radio frequencies, we selected a sample of galaxy clusters (listed in Table 1) on the basis of their clusterscale X-ray morphology and lack of evidence of a recent major merger. Cuciti et al. (2015) and Cassano et al. (2016) studied a sample of clusters selected from the Planck Sunyaev-Zel'dovich catalogue (PSZ; Planck Collaboration XXIX 2014) with a mass of $M_{500} \geq 6 \times 10^{14} M_{\odot}$ in the redshift range ${ }^{2} 0.08<z<0.33$ and $0.2<z<0.33$, respectively. For this study, we selected the clus-

\footnotetext{
2 The sample in Cuciti et al. (2015) consists of clusters with available radio and X-ray data. The sample in Cassano et al. (2016), although covering a smaller range in redshift, also includes clusters with available
}

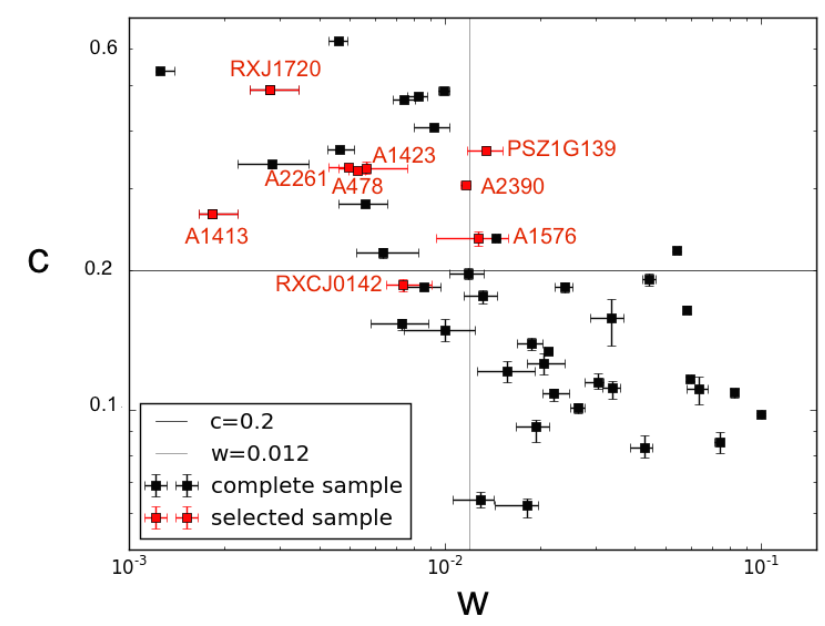

Fig. 1. Diagram of the X-ray morphological indicators based on Chandra observations for the galaxy clusters, the emission centroid shift, $w$, and the concentration parameter, $c$, of the mass-selected cluster sample in Cuciti et al. (2015) and Cassano et al. (2016). Red squares indicate the sources of our sample. Following Cassano et al. (2010), we adopted the values $w \leq 0.012$ and $c \geq 0.2$ as an indication of the distinction between merging/non-merging clusters. Merging clusters lie in the bottom right region of the plot, whilst non-merging clusters in the top left region.

ters whose dynamical status has been classified as non-merging from Chandra X-ray observations. In particular, we focus on two morphological indicators: the concentration parameter and centroid shift, which have been shown to be the most sensitive to the cluster dynamical state Lovisari et al. (2017), We briefly describe these two parameters, as follows:

- the concentration parameter, $c$, is defined as the ratio of the $\mathrm{X}$-ray surface brightness within $100 \mathrm{kpc}$ over the X-ray surface brightness within $500 \mathrm{kpc}$. It helps to select clusters with a compact core, i.e. clusters whose core has not been disrupted by a merger (Santos et al. 2008),

$$
c=\frac{S(<100 \mathrm{kpc})}{S(<500 \mathrm{kpc})}
$$

- and the emission centroid shift, $w$, defined as the standard deviation of the projected separation $\Delta$ between the peak and the cluster X-ray centroid computed within $N$ circles of increasing radius $\mathrm{R}$ (e.g. Böhringer et al. 2010),

$$
w=\frac{1}{R} \times \sqrt{\frac{\sum_{i=0}^{N}\left(\Delta_{i}-\langle\Delta\rangle\right)^{2}}{N-1}} .
$$

High values of $w$ indicate a dynamically disturbed system, while high values of $c$ indicate a peaked core that is typical of nonmerging systems. We selected the clusters labelled as "relaxed" in Cassano et al. (2016) and Cuciti et al. (2015). In addition, we added the constraint that clusters must be easily observed with LOFAR, i.e. with declination greater than $10^{\circ}$. The selected clusters, which are shown as red symbols in Fig. 1 (together with the clusters belonging to the complete sample), lie in the top left region of the $c-w$ plot (non-merging clusters). We note that many of these clusters lie near the merging/non-merging boundary (defined with the values $w \sim 0.012$ and $c \sim 0.2$ in Cassano et al. (2010), where $w$ is derived at $500 \mathrm{kpc}$ ). These

$\mathrm{X}$-ray data but without radio observations. In total, a completeness in mass greater than $80 \%$ is achieved. 
Table 1. Observation details of the targets.

\begin{tabular}{|c|c|c|c|c|c|c|c|}
\hline Name & $\begin{array}{c}\text { RA } \\
(\mathrm{h} \mathrm{m} \mathrm{s}, \mathrm{J} 2000) \\
\end{array}$ & $\begin{array}{c}\text { Dec } \\
\left({ }^{\prime \prime \prime \prime}, \mathrm{J} 2000\right) \\
\end{array}$ & $\begin{array}{c}\text { LoTSS obs. } \\
\text { ID }\end{array}$ & $\begin{array}{c}\text { LoTSS obs. } \\
\text { date }\end{array}$ & $\begin{array}{l}\text { Radio flux } \\
\text { calibrator }\end{array}$ & $\begin{array}{l}\text { Chandra obs. } \\
\text { ID }\end{array}$ & $\begin{array}{c}\text { Chandra clean } \\
\text { exp. time (ks) }\end{array}$ \\
\hline RXCJ0142.0+2131 & 014202.6 & +213119.0 & LC9_011 (P) & $21-02-18$ & 3C196 & 10440 & 19.9 \\
\hline A478 & 041320.7 & +102835.0 & LC8_006 (P) & 7/21-11-17 & 3C196 & $\begin{array}{c}1669,602,6928 \\
6929,7217,7128 \\
7222,7231,7232 \\
7233,7234,7235\end{array}$ & 139.3 \\
\hline PSZ1G139.61+24 & 062204.6 & +744051.6 & LC8_022 & $27-07-17$ & $3 \mathrm{C} 295$ & 15139,15297 & 23.1 \\
\hline A1413 & 115518.9 & +232431.0 & LC9_020 (P) & $25-01-18$ & $3 C 295$ & $\begin{array}{c}537,1661,5002 \\
5003,7696\end{array}$ & 127.8 \\
\hline A1423 & 115722.5 & +33 3918.0 & LC8_022 & 21-09-17 & 3C196 & 528,11724 & 33.3 \\
\hline A1576 & 123759.0 & +631126.0 & LT5_007 & 04-04-16 & $3 \mathrm{C} 196$ & 7938,15127 & 28.5 \\
\hline RXJ1720.1+2638 & 172010.1 & +263729.5 & LC7_004 (P) & $25-01-17$ & $3 \mathrm{C} 295$ & $1453,3224,4361$ & 42.3 \\
\hline A 2261 & 172227.1 & +320802.0 & LC6_015 & $27-07-17$ & $3 \mathrm{C} 295$ & 550,5007 & 30.6 \\
\hline A 2390 & 215334.6 & +174011.0 & LC9_030 & $18-12-17$ & 3C196 & $500,501,4193$ & 99.1 \\
\hline
\end{tabular}

Notes. The letter $\mathrm{P}$ indicates the clusters observed as part of dedicated proposals.

Table 2. Properties and literature information of the selected sample of non-merging massive clusters.

\begin{tabular}{ccccccccc}
\hline \hline Name & $z$ & $\begin{array}{c}\text { Scale } \\
\left(\mathrm{kpc} /{ }^{\prime \prime}\right)\end{array}$ & $\begin{array}{c}M_{500} \\
\left(10^{14} M_{\odot}\right)\end{array}$ & $\begin{array}{c}K_{0} \\
\left(\mathrm{keV} \mathrm{cm}^{2}\right)\end{array}$ & X-ray & Radio & Telescope \& frequency & Ref. \\
\hline RXCJ0142.0+2131 & 0.280 & 4.28 & 6.07 & 131 & nCC & UL & GMRT: $235 / 610 \mathrm{MHz}$ & 1 \\
A478 & 0.088 & 1.66 & 7.06 & 8 & CC & MH & VLA: $1.4 \mathrm{GHz}$ & 2 \\
PSZ1G139.61+24 & 0.270 & 4.17 & 7.09 & 10 & CC & cMH & GMRT: $610 \mathrm{MHz}$ & 3 \\
A1413 & 0.143 & 2.53 & 5.98 & 64 & nCC & cMH & VLA: $1.4 \mathrm{GHz}$ & 4 \\
A1423 & 0.214 & 3.50 & 6.09 & 27 & CC & UL & GMRT: $610 \mathrm{MHz}$ & 5 \\
A1576 & 0.302 & 4.51 & 5.98 & 186 & nCC & UL & GMRT: $610 \mathrm{MHz}$ & 1 \\
RXJ1720.1+2638 & 0.164 & 2.84 & 6.34 & 21 & CC & MH & GMRT: $317 / 617 \mathrm{MHz}, 1.28 \mathrm{GHz}$ & 6 \\
& & & & & & & VLA: $1.5,4.9,8.4 \mathrm{GHz}$ & 6 \\
A2261 & 0.224 & 3.63 & 7.39 & 61 & nCC & H & VLA: $1.4 \mathrm{GHz}$ & 7 \\
A2390 & 0.234 & 3.75 & 9.48 & 15 & CC & H & VLA: $1.4 \mathrm{GHz}$ & 7 \\
\hline
\end{tabular}

Notes. Column 1: name of the cluster; Col. 2: redshift; Col. 3: angular to physical scale conversion at the cluster redshift; Col. 4: mass within the radius enclosing a mean density of 500 times the critical density (Planck Collaboration XXIX 2014); Col. 5: core entropy from X-ray data (Giacintucci et al. 2017); Col. 6: presence of a cool core (CC) from X-ray data (Giacintucci et al. 2017); Col. 7: presence of radio diffuse emission as present in the literature ( $\mathrm{UL}=$ upper limit; $\mathrm{MH}=$ mini halo; $\mathrm{cMH}=$ candidate mini halo; $\mathrm{H}=$ halo); Col. 8: observations present in the literature; Col. 9: radio references.

References. (1) Kale et al. 2013; (2) Giacintucci et al. 2014b; (3) Giacintucci et al. 2017; (4) Govoni et al. 2009 (5) Venturi et al. 2008; (6) Giacintucci et al. 2014a; (7) Sommer et al. 2017.

clusters are more likely to be slightly disturbed systems, thus through their observations we can search for low-frequency steep-spectrum emission powered by minor/off-axis mergers.

\section{Data reduction}

\subsection{Radio: LOFAR observations}

Five of the targets were observed as part of the deep imaging survey LoTSS (LOFAR Two-metre Sky Survey; Shimwell et al. 2017). The remaining four were observed as part of dedicated proposals (LC7_004, LC8_006, LC9_011, LC9_020), adopting the LoTSS observing set-up ${ }^{3}$. The observations were carried out at $120-168 \mathrm{MHz}$ using the high band antenna (HBA) with a total

\footnotetext{
3 We note that the clusters observed for the proposals lie at the centre of the pointings and the clusters observed within LoTSS lie at a maximum distance from the phase centre of the pointing of $1^{\circ}$, where the primary beam response is $>0.8$. This allows us to reach a consistent sensitivity to diffuse emission for the whole sample.
}

on-source time of $8 \mathrm{~h}$ preceded and followed by a flux density calibrator observation for $10 \mathrm{~min}$. More details can be found in Table 1 . The calibration and imaging procedure is based on the facet calibration scheme presented in van Weeren et al. (2016b). A complete outline of the procedure can be found in Savini et al. (2018b). The main steps are as follows:

- Preliminary pre-processing, which was performed by the Radio Observatory (ASTRON) and has been applied to the data;

- Direction-independent calibration, which was obtained by executing the Prefactor pipeline ${ }^{4}$, following the strategy outlined in de Gasperin et al. (2019);

- Direction-dependent calibration using the FACTOR pipeline $^{5}$.

Each field of view was divided into a discrete number of facets that are separately calibrated. The calibrator for each facet was

\footnotetext{
4 https://github.com/lofar-astron/prefactor

5 https://github.com/lofar-astron/factor
} 
selected with a minimum flux density specified by the user in a range between $0.3 \mathrm{Jy}$ and $0.6 \mathrm{Jy}$. The facets are usually processed in order of calibrator flux density, before processing those bordering the cluster facet. The cluster facet is the last to be processed, so that it could benefit from the calibration of the preceding facets. The facet images are stitched together to form a mosaic and the mosaicked final image is corrected for the primary beam. In line with other LOFAR HBA studies, we adopted a systematic calibration error of $15 \%$ on all the measured flux densities (Shimwell et al. 2016, van Weeren et al. 2016a, Savini et al. 2018b). Radio imaging was performed through the WSClean package (Offringa et al. 2014) implemented in FACTOR, varying the robust values of the Briggs weighting (Briggs 1995) and $u v$-taper to obtain different resolutions and increase the sensitivity to diffuse emission. To obtain specific images, such as spectral index maps, compact-source-subtracted images, and halo-injected images, we used the Common Astronomy Software Applications (CASA, version 4.5.2; McMullin et al. 2007) with the multi-scale option of the clean task and took the $w$-projection parameter into account.

\subsection{Radio: GMRT observation}

We processed archival GMRT observations at $610 \mathrm{MHz}$ of the clusters A478 and RXJ1720.1+2638 using the Source Peeling and Atmospheric Modeling (SPAM) pipeline (see Intema et al. 2017 for details) to perform a detailed spectral analysis with the LOFAR observations. A478 and RXJ1720.1+2638 were observed, respectively, on October 10, 2011 (under project code 21_017) and July 24, 2011 (under project code 11MOA01). Visibilities at $610 \mathrm{MHz}$ were recorded in one polarisation (RR) over a bandwidth of $32 \mathrm{MHz}$, as part of a dual-frequency observation. The on-source time was $3.4 \mathrm{~h}$ for $\mathrm{A} 478$ and $5.2 \mathrm{~h}$ for RXJ1720.1+2638. The primary calibrator used for flux and bandpass calibrations in both observations was $3 \mathrm{C} 48$. We adopted the same flux standard as for LOFAR (Scaife \& Heald 2012). A $T_{\text {sys }}$ gain correction of 0.963 for A478 and 0.981 for RXJ1720.1+2638 was derived using the all-sky map at $408 \mathrm{MHz}$ by Haslam et al. (1995); this $T_{\text {sys }}$ gain correction was subsequently applied. The pipeline removed $56 \%$ of the data of A478 due to Radio Frequency Interferences (RFI) and various telescope issues and the pipline removed $48 \%$ for RXJ1720.1+2638. The pipeline output visibilities were imported into CASA for final imaging, using the multi-scale option of the clean task. Our highest fidelity images reach a sensitivity of $65 \mu \mathrm{Jy}$ beam $^{-1}$ with a $4.8^{\prime \prime} \times 3.9^{\prime \prime}$ beam and $45 \mu \mathrm{Jy}$ beam $^{-1}$ with a $5.0^{\prime \prime} \times 4.8^{\prime \prime}$ beam, for A478 and RXJ1720.1+2638 respectively. We adopted a $10 \%$ scale error on all flux density measurements (Chandra et al. 2004).

\subsection{X-ray: Chandra observation}

To investigate the connection between the thermal and nonthermal components in the ICM, we reprocessed Chandra $\mathrm{X}$-ray observations for each cluster in the sample. The ID and clean exposure time of each observation can be found in Table 1. We carried out a standard data reduction using CIAO v4.9 and Chandra CALDB v4.7.3 to produce the exposure-corrected images in the $0.5-2.0 \mathrm{keV}$ band shown in the paper (see Botteon et al. 2018 for the procedure outline).

The Chandra X-ray density and temperature profiles of the clusters we selected were already obtained and combined by Giacintucci et al. (2017) to derive the specific entropy at the cluster centre, $K_{0}$. We used the values of $K_{0}$ for the clusters in our sample to distinguish between cool-core or non-cool-core clusters following Giacintucci et al. (2017): clusters with low central entropies $\left(K_{0}<30-50 \mathrm{keV} \mathrm{cm}^{2}\right)$ are expected to host a cool core. The values relevant for our sample are reported in Table 2, where we specify whether the clusters host a cool core or not, according to the above classification.

\section{Results}

In the following, each cluster is described in a separate subsection. The information from the literature is summarised in Table 2, while the LOFAR results are summarised in Table 3. In Figs. 2-12, we present the images obtained at low frequencies in our campaign of LOFAR observations: a high- and a lowresolution image at the central frequency of $144 \mathrm{MHz}$ for each cluster and overlays with the X-ray images taken by Chandra and optical images taken within the Sloan Digital Sky Survey (SDSS) or the Panoramic Survey Telescope and Rapid Response System (Pan-STARRS). Our high-resolution LOFAR images with a larger field of view, which show the quality of the facet calibration and the presence of additional sources in the cluster periphery, can be found in the Appendix. The size of the diffuse emission is given by $D_{\text {radio }}=\sqrt{D_{\min } \times D_{\max }}$, where $D_{\min }$ and $D_{\max }$ are the minimum and maximum diameter of the $3 \sigma$ surface brightness isocontours (e.g. Cassano et al. 2008; Giacintucci et al. 2017), as measured from the low-resolution LOFAR images.

In some cases, to reveal diffuse emission, it is necessary to subtract the contribution of compact sources in the cluster centre. Therefore, we image the sources at high resolution applying a cut in the $u v$-range, subtract them from the $u v$-data, and reimage the data sets at lower resolution (see Wilber et al. (2018) for details on the subtraction procedure). The value of the $u v$-cut varies from target to target, depending on the extension of the sources that we attempt to subtract. The error on the total flux density is computed as $\sqrt{\sigma_{\text {cal }}^{2}+\sigma_{\text {sub }}^{2}}$, where $\sigma_{\text {cal }}$ is equal to $15 \%$ of the measured flux density and $\sigma_{\text {sub }}$ is the subtraction error. The latter is derived by varying the range of the $u v$-cut that is used for modelling the compact sources. We can then quantify the error on the total flux density of the diffuse emission after subtracting different models.

When no hint of cluster-scale radio emission is detected, we provide upper limits on the radio power through the mock halo injection procedure (e.g. Venturi et al. 2008, Bonafede et al. 2017). We created a mock radio halo with a central brightness $I_{0}$ and an $e$-folding radius $r_{\mathrm{e}}$ derived from the total radio power at $1.4 \mathrm{GHz}\left(P_{1.4}\right)$ and halo size $\left(R_{\mathrm{H}}\right)$ following the known powermass correlation found by Cassano et al. (2013) for haloes. We used the relation $R_{\mathrm{H}} / r_{\mathrm{e}}=2.6$ found by Bonafede et al. (2017), who compared the values of $R_{\mathrm{H}}$ and $r_{\mathrm{e}}$ of the clusters; these values are both in the samples of Cassano et al. (2007) and Murgia et al. (2009). We then carried out a Fourier transform of the mock source into the $u v$-data of the LOFAR observation, which is then imaged taking into account the $w$-projection parameter. We chose a relatively empty region near the cluster centre, void of bright sources or artefacts, to host the injected flux density. We decreased the total flux density of the mock halo until it could not be detected in the LOFAR image, i.e. when the surface brightness above $2 \sigma$ has a maximum linear size $<3 r_{\mathrm{e}}$ following Bonafede et al. (2017) and Wilber et al. (2018). Assuming the typical spectral index value for the haloes $(\alpha=-1.3)$, we then rescaled the total flux density of the mock halo to $1.4 \mathrm{GHz}$ and computed the limit to the total radio power at $1.4 \mathrm{GHz}$, which can be compared with values present in the literature. 
F. Savini et al.: A LOFAR study of non-merging massive galaxy clusters

Table 3. Derived physical parameters of the radio diffuse emission of our target clusters as seen by LOFAR.

\begin{tabular}{|c|c|c|c|c|c|}
\hline Name & LOFAR class. & $\begin{array}{c}D_{\text {radio }} \\
(\mathrm{kpc})\end{array}$ & $\begin{array}{l}S_{144}^{\text {diff }} \\
(\mathrm{mJy})\end{array}$ & $\begin{array}{c}P_{144}^{\mathrm{diff}} \\
\left(\times 10^{24} \mathrm{~W} \mathrm{~Hz}^{-1}\right)\end{array}$ & Notes \\
\hline RXCJ0142.0+2131 & $\mathrm{H}$ & 570 & $32 \pm 6$ & $8.6 \pm 1.6$ & New H discovered \\
\hline $\mathrm{A} 478$ & uncertain & - & - & - & No diffuse emission \\
\hline PSZ1G139.61+24 & $\mathrm{MH}+\mathrm{USSH}$ & 550 & $30 \pm 4$ & $7 \pm 1$ & MH: $3 \times 10^{24} \mathrm{~W} \mathrm{~Hz}^{-1}$, USSH: $4 \times 10^{24} \mathrm{~W} \mathrm{~Hz}^{-1}$ \\
\hline A1413 & $\mathrm{MH}$ & 210 & $40 \pm 7$ & $2.3 \pm 0.4$ & MH confirmed \\
\hline A 1423 & UL & - & 23 & 3.3 & Mock H injection; $P_{1.4}<0.17 \times 10^{24} \mathrm{~W} \mathrm{~Hz}^{-1}(\alpha=-1.3)$ \\
\hline A1576 & $\mathrm{UL}$ & - & 37 & 11.9 & Mock H injection; $P_{1.4}<0.62 \times 10^{24} \mathrm{~W} \mathrm{~Hz}^{-1}(\alpha=-1.3)$ \\
\hline RXJ1720.1+2638 & $\mathrm{MH}+\mathrm{USSH}$ & 600 & $820 \pm 123$ & $72 \pm 11$ & MH: $64 \times 10^{24} \mathrm{~W} \mathrm{~Hz}^{-1}, \mathrm{USSH}: 8 \times 10^{24} \mathrm{~W} \mathrm{~Hz}^{-1}$ \\
\hline A2261 & $\mathrm{H}$ & 1200 & $\sim 165$ & $\sim 26$ & H confirmed \\
\hline A2390 & uncertain & 1100 & - & - & $600 \mathrm{kpc}$-central double radio galaxy \\
\hline
\end{tabular}

Notes. Column 1: name of the cluster; Col. 2: classification of the diffuse emission as based on observations at $144 \mathrm{MHz}$ (UL= upper limit; $\mathrm{MH}=$ mini halo; $\mathrm{cMH}=$ candidate mini halo; $\mathrm{H}=$ halo; $\mathrm{USSH}=\mathrm{USS}$ halo); Col. 3: size of the diffuse emission as measured from the low-resolution LOFAR images; Col. 4: total flux density at $144 \mathrm{MHz}$; Col. 5: total radio power at $144 \mathrm{MHz}$; Col. 6: notes on the final image.

\subsection{RXCJ0142.0+2131}

The non-cool-core cluster RXCJ0142.0+2131 is known to host a radio source at the cluster centre, that is coincident with the peak of the X-ray emission. Several bright radio sources in the field are visible in the image of the cluster (Kale et al. 2013). The X-ray distribution has a relatively disturbed morphology with no strong features. We note that this cluster exhibits the smallest value of $c$ in the sample.

Based on optical data, the occurrence of a possible merger event was inferred (Barr et al. 2006), considering the presence of the second BCG (to the north-east) at $z=0.283$ located at $\sim 650 \mathrm{kpc}$ from the cluster centre and the X-ray morphology elongated in the same direction (von der Linden et al. 2014).

Since no diffuse radio emission has been detected in this cluster, an upper limit on the radio power was derived by Kale et al. (2013) using GMRT observations at $610 \mathrm{MHz}$ and $235 \mathrm{MHz}$. The upper limit rescaled at $1.4 \mathrm{GHz}$ is $P_{1.4} \leq 0.45 \times$ $10^{24} \mathrm{~W} \mathrm{~Hz}^{-1}$ (Cassano et al. 2013).

The high-resolution LOFAR image we obtained (left panel of Fig. 2) is consistent with the $610 \mathrm{MHz}$ GMRT image in Kale et al. (2015) with a central radio source and few other radio sources to the north-east and south-west. We subtracted the contribution of the sources detected at high resolution using only the longer baselines with a $u v$-cut of $>1750 \lambda$ (that corresponds to $500 \mathrm{kpc}$ ) and re-imaged the data at lower resolution to search for diffuse emission. We were then able to see the presence of centrally located diffuse emission with $D_{\text {radio }} \sim 570 \mathrm{kpc}$, as shown in the right panel of Fig. 2. The total flux density is $32 \pm 6 \mathrm{mJy}$ corresponding to a total radio power at $144 \mathrm{MHz}$ of $(8.6 \pm 1.6) \times 10^{24} \mathrm{~W} \mathrm{~Hz}^{-1}$. Using the upper limit at $1.4 \mathrm{GHz}$ placed by Kale et al. (2013), we can estimate the spectral index to be $\alpha_{144}^{1400}<-1.3$. We note that this is a conservative estimate, since the upper limit is computed considering a $1 \mathrm{Mpc}$ halo, while the halo we detect is on a scale of less than $600 \mathrm{kpc}$. We can use the power-luminosity plot in Fig. 4 in Brunetti et al. (2007), where upper limits for $500 \mathrm{kpc}$-haloes are also derived for the typical GMRT observations of the radio halo survey. At the redshift of this cluster, a limit evaluated on a size of $500 \mathrm{kpc}$ would be 1.6-1.8 times deeper than that on a size of $1 \mathrm{Mpc}$. Hence, we derive a more stringent limit on the spectrum of $\alpha_{144}^{1400}<-1.6$, which would lead to an USS halo classification.

The size, although smaller than that of giant haloes, and the estimated spectral index value are consistent with the properties of a radio halo, likely an USS halo. We note that the power measured with LOFAR and extrapolated at $1.4 \mathrm{GHz}$ lies below the known power-mass correlation of Cassano et al. (2013; see Fig. 14).

\section{2. $A 478$}

A478 is a cool-core cluster with a relaxed X-ray morphology and a spherically symmetric temperature distribution on large scales. A number of X-ray substructures at the cluster centre, such as small cavities with sizes of few kpc, are found by Sun et al. (2003). Giacintucci et al. (2014b) presented VLA radio images at $1.4 \mathrm{GHz}$ and reveal the presence of a central double-lobe radio galaxy with a size of $\sim 13 \mathrm{kpc}$. The hosting galaxy is the BCG, as can be seen in the optical overlay. Diffuse, low-brightness radio emission encompassing the central active galactic nucleus (AGN) and extending on a scale of $\sim 300 \mathrm{kpc}$ is also detected and classified as a mini halo. The low-resolution image at $1.4 \mathrm{GHz}$ (Fig. 1c in Giacintucci et al. 2014b) shows that the mini halo blends with the tail of a cluster-member head-tail radio galaxy located to the north-east of the cluster centre. The tail extends for $\sim 200 \mathrm{kpc}$ and encompasses an unresolved source, which is also a cluster member. A second unresolved source to the north with no optical identification is thought to be a background galaxy.

Markevitch et al. (1995) reported the presence of a cold front in the Chandra image at $\sim 60 \mathrm{kpc}$ to the south-west of the cluster, which we show in the X-ray image of Fig. 3. Its position seems to indicate that the radio emission is confined by the front, as expected in simulations of mini haloes (ZuHone et al. 2013).

The calibration of the LOFAR data set was difficult owing to the presence of a $3 \mathrm{C}$ source $(3 \mathrm{C} 109$; $04 \mathrm{~h} 13 \mathrm{~m} 40.37 \mathrm{~s}$, $\left.+11^{\circ} 12^{\prime} 13.8^{\prime \prime}\right)$ with a flux density of $\sim 21 \mathrm{Jy}$ located at $0.7^{\circ}$ from the target. We created a sky model of this double-lobe radio galaxy using a high-resolution VLA image at $4.8 \mathrm{GHz}$ to improve its calibration. However, the image sensitivity is limited by the dynamic range, therefore we consider the caveats relative to the presence of negative holes around the central source, and the higher noise of the target facet $\left(460 \mu \mathrm{Jy} \mathrm{beam}^{-1}\right)$ compared to that obtained for the other clusters. Nevertheless, the final image of A478 at $144 \mathrm{MHz}$ (Fig. 3) confirms the presence of the central AGN; the head-tail radio galaxy to the north-east with a tail is overall much longer than previously seen, extending for more than $650 \mathrm{kpc}$. Interestingly, the tail appears to be divided into two regions. This is seen in the case of A1033 de Gasperin et al. (2017), in which the authors have proposed the gentle re-energisation of electrons as the mechanism that 

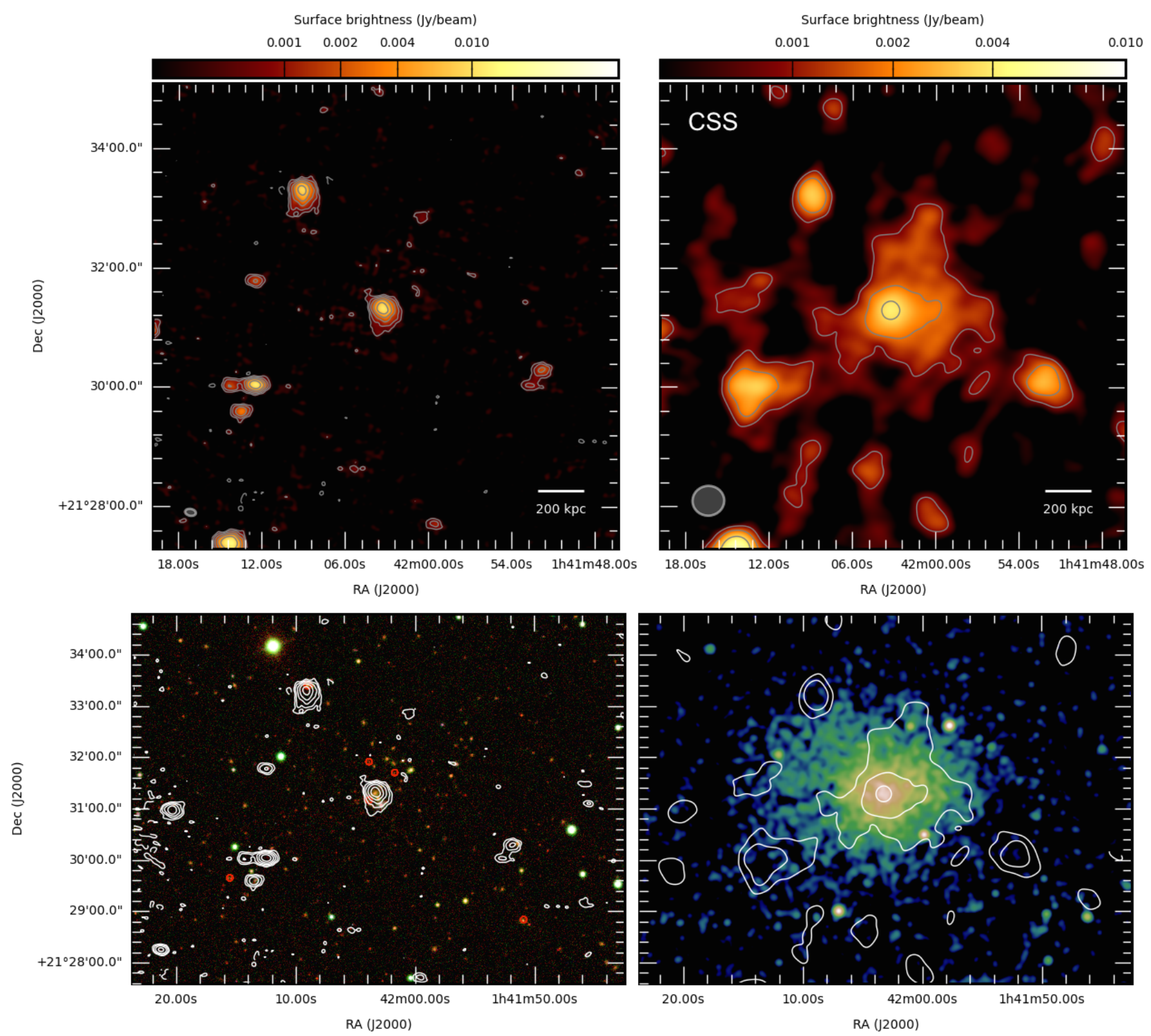

Fig. 2. RXCJ0142.0+2131. Top left panel: high-resolution $144 \mathrm{MHz}$ LOFAR image of RXCJ0142.0+2131. The contour levels start at $3 \sigma$ where $\sigma=150 \mu \mathrm{Jy}_{\text {beam }}{ }^{-1}$, and are spaced by a factor of two. The negative contour level at $-3 \sigma$ is overlaid with a dashed line. The beam is $11^{\prime \prime} \times 7^{\prime \prime}$ and is shown in grey in the bottom left corner of the image. Top right panel: low-resolution $144 \mathrm{MHz}$ LOFAR image of RXCJ0142.0+2131. The contour levels start at $3 \sigma$ where $\sigma=300 \mu \mathrm{Jy}_{\text {beam }}{ }^{-1}$, and are spaced by a factor of two. The negative contour level at $-3 \sigma$ is overlaid with a dashed line. The beam is $26^{\prime \prime} \times 24^{\prime \prime}$ and is shown in grey in the bottom left corner of the image. This image was obtained after central source subtraction (CSS) with a taper of $15^{\prime \prime}$ and Briggs weighting (robust $=0$ ). Bottom left panel: optical Pan-STARRS image of RXCJ0142.0+2131 with the high-resolution $\left(11^{\prime \prime} \times 7^{\prime \prime}\right) 144 \mathrm{MHz}$ LOFAR contours overlaid. The red circles indicate the cluster-member galaxies with available spectroscopic redshift. Bottom right panel: Chandra X-ray image of RXCJ0142.0+2131 smoothed on a scale of 5" with the low-resolution $\left(26^{\prime \prime} \times 24^{\prime \prime}\right) 144 \mathrm{MHz}$ LOFAR contours overlaid.

justifies the brightness of the second part of the radio tail, which is only visible at low frequencies. With LOFAR, we do not see hints of centrally located diffuse emission. As a further argument, we injected mock mini haloes with different sizes and flux densities in the data set, both at the cluster centre and in a void close-by region, to rule out the possibility that the calibration artefacts are responsible for the absence of the mini halo (see Fig. A.1). We used the minimum and maximum values for $r_{\mathrm{e}}$, i.e. 25 and $100 \mathrm{kpc}$, and for $I_{0}$ at $1.4 \mathrm{GHz}$, i.e. 13 and $1 \mu \mathrm{Jy} \mathrm{beam}^{-1}$, respectively, found by Murgia et al. (2009) for a sample of mini haloes. Considering the flux density reported in Giacintucci et al.
(2017) for the mini halo in A478, the recovered flux density of the mock mini halo indicates that the limit on the spectral index of the source would be $\alpha>-1$, which is unusually flat for a mini halo. We processed an archival GMRT observation at $610 \mathrm{MHz}$ and obtain the low-resolution image shown in the left panel of Fig. 4. Neither diffuse emission nor the second portion of the tail is visible. We re-imaged the LOFAR and GMRT data sets with a Gaussian taper of 30" and with the same pixel size, baseline range (200-40 000 $\lambda$ ), and uniform weighting scheme to minimise the differences in the $u v$-coverage of the two observations, and we obtain the spectral index map that is shown in the 

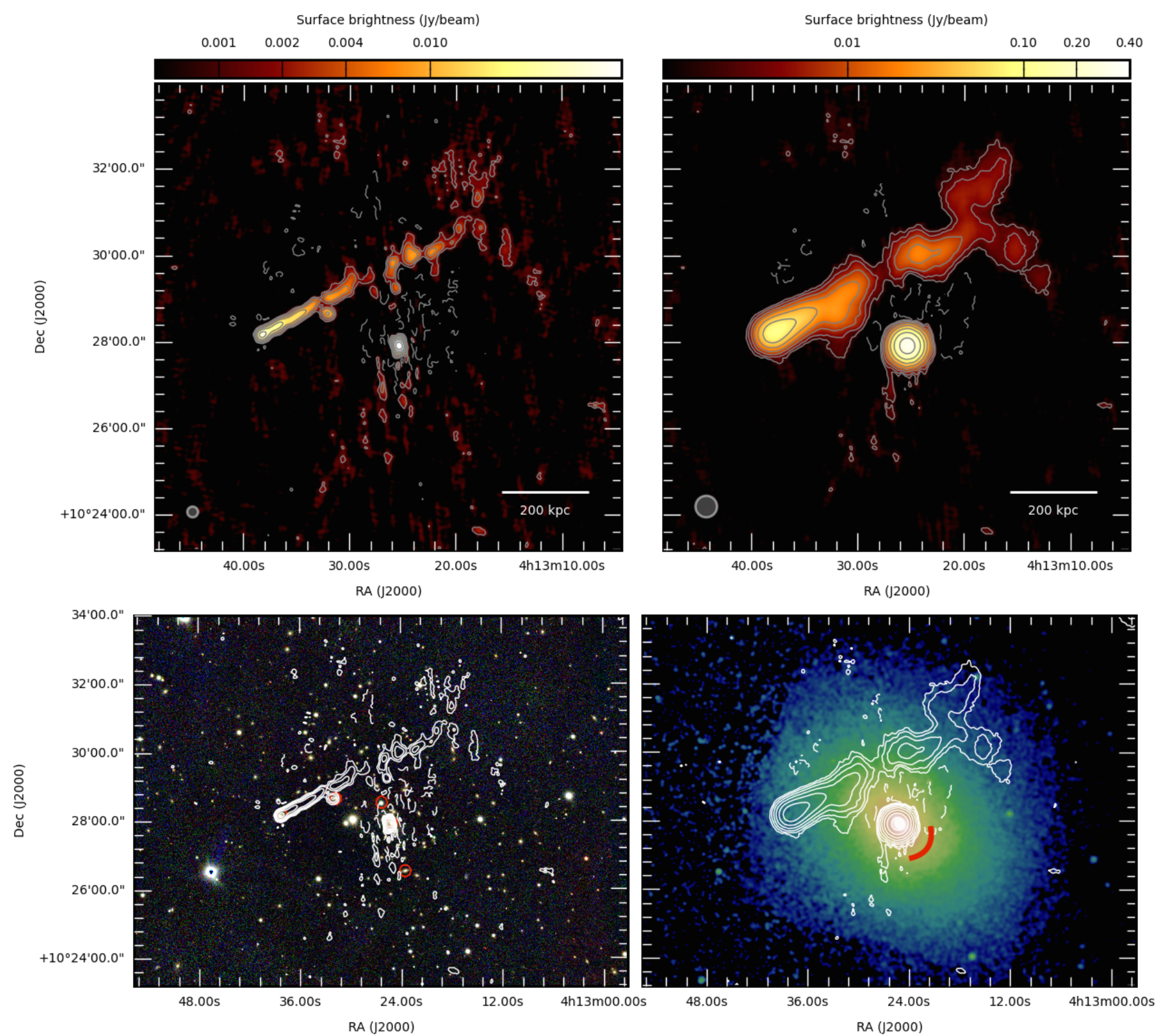

Fig. 3. A478. Top left panel: high-resolution $144 \mathrm{MHz}$ LOFAR image of A478. The contour levels start at $3 \sigma$ where $\sigma=460 \mu \mathrm{Jy}$ beam $^{-1}$ and are spaced by a factor of two. The negative contour level at $-3 \sigma$ is overlaid with a dashed line. The beam is $10^{\prime \prime} \times 10^{\prime \prime}$ and is shown in grey in the bottom left corner of the image. Top right panel: low-resolution $144 \mathrm{MHz}$ LOFAR image of A478. The contour levels start at $3 \sigma$, where $\sigma=620 \mu \mathrm{Jy}_{\mathrm{beam}}{ }^{-1}$, and are spaced by a factor of two. The negative contour level at $-3 \sigma$ is overlaid with a dashed line. The beam is $30^{\prime \prime} \times 30^{\prime \prime}$ and is shown in grey in the bottom left corner of the image. Bottom left panel: optical Pan-STARRS image of A478 with the high-resolution $\left(10^{\prime \prime} \times 10^{\prime \prime}\right)$ $144 \mathrm{MHz}$ LOFAR contours overlaid. The red circles indicate the cluster-member galaxies with available spectroscopic redshift. Bottom right panel: Chandra X-ray image of A478 smoothed on a scale of $5^{\prime \prime}$ with the low-resolution $\left(30^{\prime \prime} \times 30^{\prime \prime}\right) 144 \mathrm{MHz}$ LOFAR contours overlaid. The red arc indicates the position of the cold front found by Markevitch et al. (1995).

right panel of Fig. 4. The central emission has a spectral index of $\sim-1.1$ and the head-tail radio galaxy has the typical trend of an active radio galaxy with a flat core $\left(\alpha_{610}^{144} \sim-0.6\right)$ and a steepening along the tail up to $\alpha_{610}^{144} \sim-3$. We can provide an upper limit on the spectrum of the second portion of the tail, using the mean flux density from the LOFAR image and the rms noise from the GMRT image. This results in a spectral index $<-3.8$.

\section{3. $P S Z 1 G 139.61+24$}

The cool-core cluster PSZ1G139.61+24 has been studied in detail in a dedicated paper by Savini et al. (2018a).
Giacintucci et al. (2017) have reported the detection of a tentative mini halo with an overall source size of $\sim 100 \mathrm{kpc}$ located at the cluster centre, and the LOFAR image presented in Savini et al. (2018a) reveals new diffuse emission extending for more than $500 \mathrm{kpc}$. The radio source associated with the cluster consists of a central bright component surrounded by halo-like emission extending beyond the cool core. The two components become apparent in a spectral analysis performed between $144 \mathrm{MHz}$ LOFAR and $610 \mathrm{MHz}$ GMRT images that reveals a central component with $\alpha_{144}^{610} \sim-1.3$, whilst the largescale faint emission exhibits an USS with an upper limit of $\alpha_{144}^{610}<-1.7$. Although the cluster core has a low entropy, 

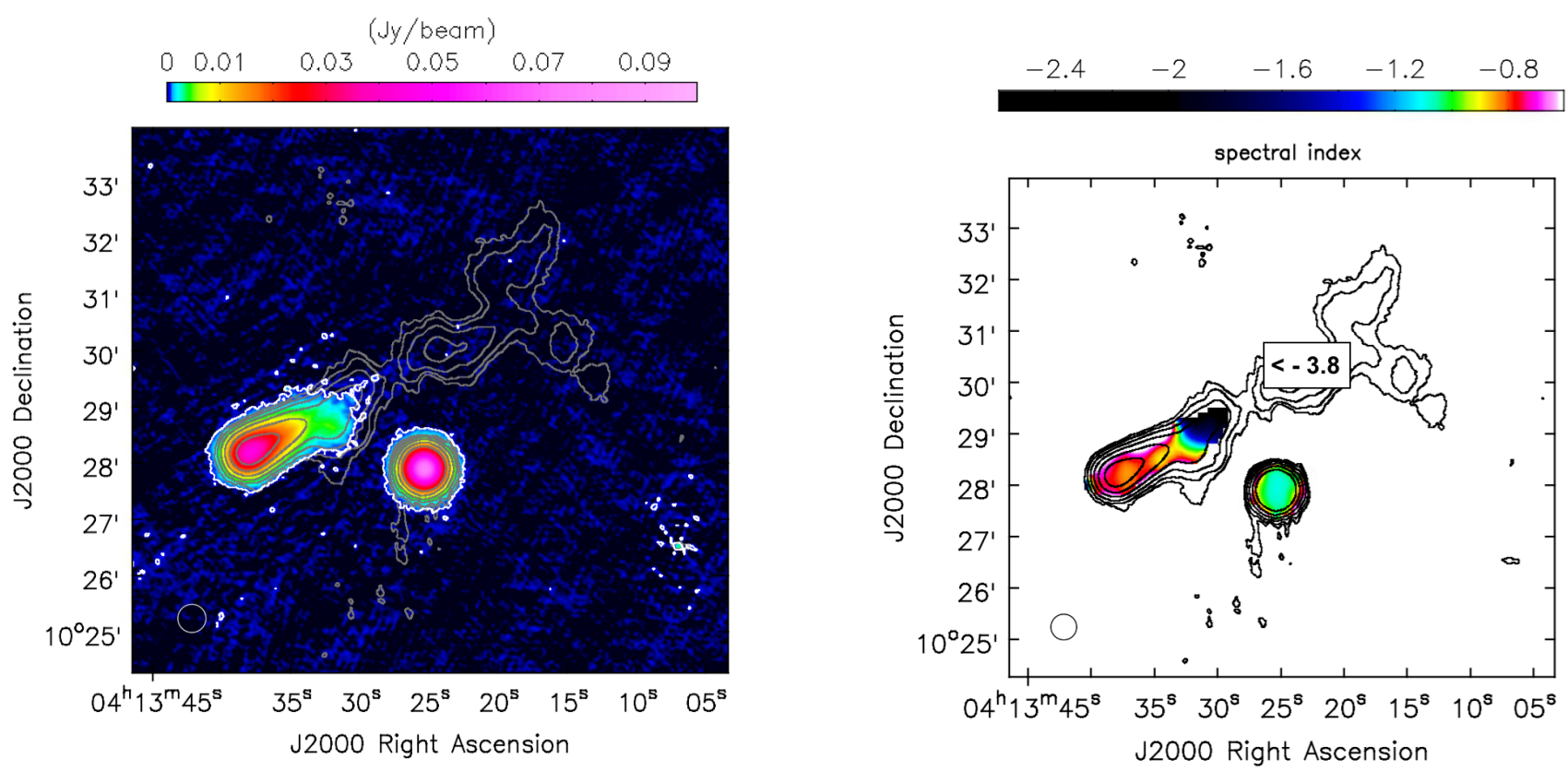

Fig. 4. Left panel: $610 \mathrm{MHz}$ GMRT image of A478 with its $3 \sigma$ contour level in white where $\sigma=90 \mu \mathrm{Jy} \mathrm{beam}^{-1}$. The beam is $30^{\prime \prime} \times 30^{\prime \prime}$ for both GMRT and LOFAR, and is shown in the bottom left corner of the image. Right panel: spectral index map between the $610 \mathrm{MHz}$ GMRT and $144 \mathrm{MHz}$ LOFAR images of A478. Contour levels from the low-resolution LOFAR image of A478 in Fig. 3 are overlaid in grey and black. Pixels below $3 \sigma$ are blanked. The error map is shown in Fig. A.2.

typical of non-merging cool-core systems, the X-ray analysis shows that the cluster is slightly disturbed and hosts a cold front (shown in the bottom panel of Fig. 5) suggesting the presence of gas sloshing. Savini et al. (2018a) have argued that the largescale radio emission outside the core is produced by a minor merger that powers electron re-acceleration without disrupting the cool core.

\section{4. $A 1413$}

The $1.4 \mathrm{GHz}$ VLA image in Govoni et al. (2009) reveals the presence of diffuse emission at the centre of the cool-core cluster A1413, extending on a scale of $\sim 220 \mathrm{kpc}$ with a total flux density of $1.9 \pm 0.7 \mathrm{mJy}$. At the sensitivity limit of the FIRST survey (Becker et al. 1995), the central optical galaxy does not contain a compact radio source (Fig. 2 in Govoni et al. 2009), and the authors suggested that the source might be a candidate mini halo, speculating about a scenario in which the central galaxy has switched off while the mini halo continues to emit. Govoni et al. (2009) also reported an offset between the emission peak of this radio source and both the central galaxy and the X-ray emission peak.

The X-ray morphology is slightly elongated in the northsouth direction and shows a bright core with a moderate entropy value $\left(K_{0}=64 \pm 8\right.$, Giacintucci et al. 2017$)$ that indicates the absence of a cool core. We note that this cluster is the only (candidate) mini halo found in a non-cool-core cluster in the sample studied in Giacintucci et al. (2017).

With the LOFAR image in Fig. 6, we confirm the presence of centrally located diffuse emission. A head-tail radio galaxy, not mentioned or shown in Govoni et al. (2009), can be seen at the west of the cluster. The optical counter part is a galaxy member at $z=0.144$. We obtain a high-resolution image using only the longer baselines with a $u v$-cut of $>2580 \lambda$ (corresponding to $\sim 200 \mathrm{kpc}$ ) to model the central compact sources (shown in the box within the top left panel of Fig. 6). We note that two sources are actually visible in the central region, one of which is co-located with the X-ray centre, hence no offset is present. We then subtract the compact sources, imaging the central radio diffuse emission that is shown in the low-resolution image of Fig. 6; we classify this source as a mini halo. The size of the diffuse emission is $\sim 210 \mathrm{kpc}$ with a total flux density of $40 \pm 7 \mathrm{mJy}$ at $144 \mathrm{MHz}$. The implied spectral index value is $\alpha_{144}^{1400} \sim-1.3$. We note that the spectral index might be steeper, considering that the flux reported by Govoni et al. (2009) is likely overestimated, since the authors subtracted only the contribution of one of the central sources, i.e. that visible at $1.4 \mathrm{GHz}$ with FIRST.

\section{5. $A 1423$}

No hint of diffuse radio emission has ever been observed in the cool-core cluster A1423 and no radio images are available in the literature. An upper limit on the radio power was derived by Venturi et al. (2008) using a GMRT observation at $610 \mathrm{MHz}$. The upper limit rescaled at $1.4 \mathrm{GHz}$ is $P_{1.4} \leq 0.38 \times 10^{24} \mathrm{~W} \mathrm{~Hz}^{-1}$ (Cassano et al. 2013).

Our high-resolution LOFAR image of A1423 (Fig. 7) shows a bright central radio source $(\sim 0.38 \mathrm{Jy})$ that is likely connected to the BCG visible in the optical image. This radio source is elongated in the north-south direction with a tail extending to the north for $\sim 400 \mathrm{kpc}$, which might be remnant emission connected to the central source. A second tailed source is found to the north-west of the cluster centre and might be connected to an optical galaxy of unknown redshift. Subtracting the bright and extended central source was not possible since it would leave residuals that cannot be distinguished from diffuse emission. The low-resolution LOFAR observation does not show additional emission. Moreover, no spatial correlation between radio and X-ray emission is found since the X-ray morphology appears disturbed along the east-west axis, whilst the radio emission is elongated along the north-south axis. 

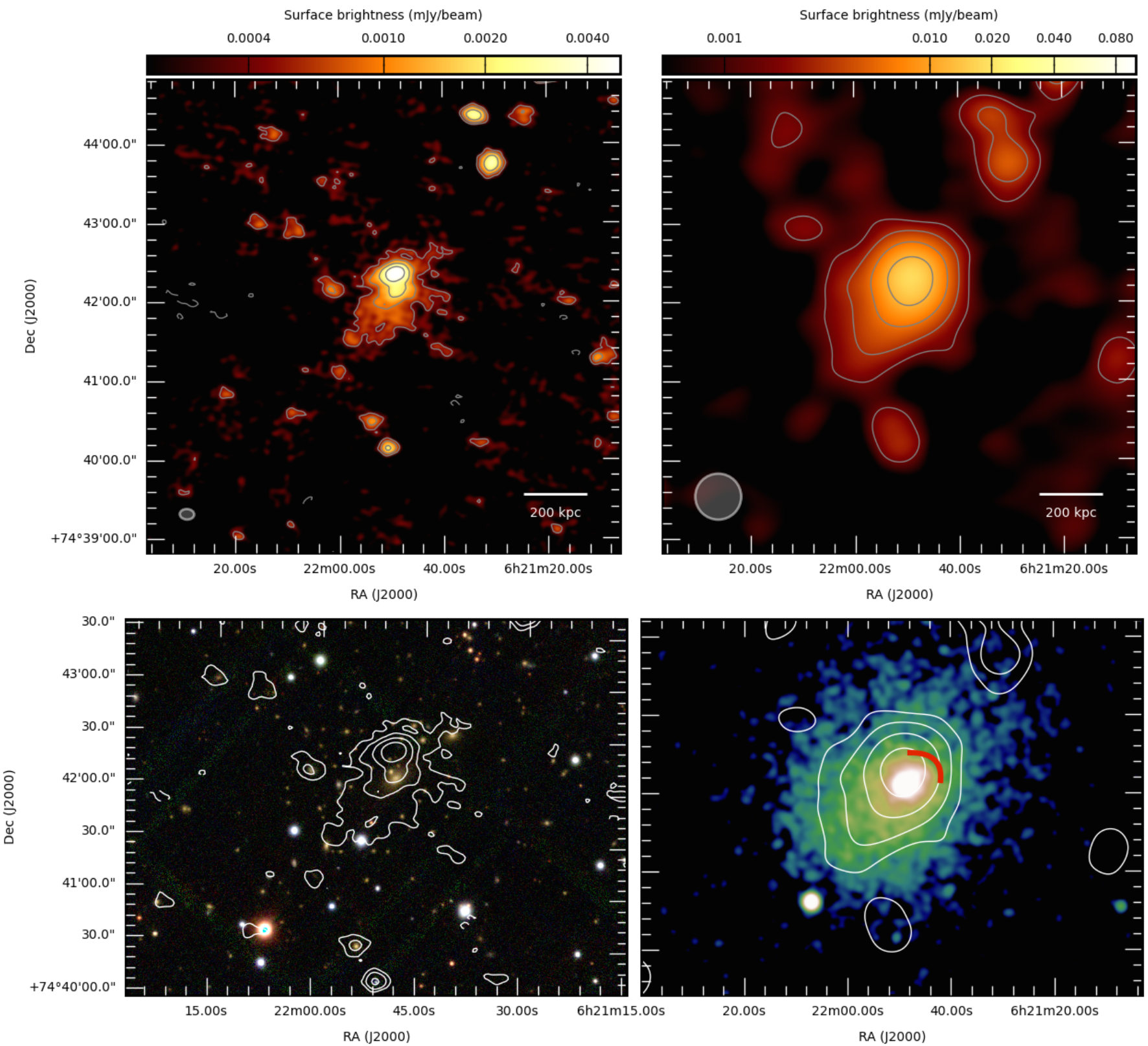

Fig. 5. PSZ1G139.61+24. Top left panel: high-resolution $144 \mathrm{MHz}$ LOFAR image of PSZ1G139.61+24. The contour levels start at $3 \sigma$, where $\sigma=150 \mu \mathrm{Jy}_{\mathrm{beam}}{ }^{-1}$, and are spaced by a factor of two. The negative contour level at $-3 \sigma$ is overlaid with a dashed line. The beam is $11^{\prime \prime} \times 8^{\prime \prime}$ and is shown in grey in the bottom left corner of the image. Top right panel: low-resolution $144 \mathrm{MHz}$ LOFAR image of PSZ1G139.61+24. The contour levels start at $3 \sigma$, where $\sigma=500 \mu \mathrm{Jy}_{\text {beam }}{ }^{-1}$, and are spaced by a factor of two. The negative contour level at $-3 \sigma$ is overlaid with a dashed line. The beam is $35^{\prime \prime} \times 35^{\prime \prime}$ and is shown in grey in the bottom left corner of the image. Bottom left panel: optical Pan-STARRS image of PSZ1G139.61+24 with the high-resolution $\left(11^{\prime \prime} \times 8^{\prime \prime}\right) 144 \mathrm{MHz}$ LOFAR contours overlaid. Bottom right panel: Chandra X-ray image of PSZ1G139.61+24 smoothed on a scale of 6 " with the low-resolution $\left(35^{\prime \prime} \times 35^{\prime \prime}\right) 144 \mathrm{MHz}$ LOFAR contours overlaid. The red arc indicates the position of the cold front found by Savini et al. (2018a).

We computed a new upper limit on the radio power by injecting a mock halo in the data set (see Sect. 4). The integrated flux density of the mock halo computed within a region centred on the injected halo with a radius equal to $R_{\mathrm{H}}=436 \mathrm{kpc}$ is $28 \mathrm{mJy}$ at $144 \mathrm{MHz}$, which corresponds to a total radio power at $144 \mathrm{MHz}$ of $P_{144}=4.1 \times 10^{24} \mathrm{~W} \mathrm{~Hz}^{-1}$. Assuming the typical spectral index value used in the power-mass correlation for haloes $(\alpha=-1.3)$, we derive a new upper limit to the radio power at $1.4 \mathrm{GHz}$, which we plot in Fig. 14. The new upper limit is $P_{1.4}<0.20 \times 10^{24} \mathrm{~W} \mathrm{~Hz}^{-1}$, i.e. almost a factor of 2 deeper than that derived in literature.

\section{6. $A 1576$}

As in the case of A1423, no hint of diffuse radio emission has so far been observed in non-cool-core cluster A1576. A central radio galaxy with indications of a jet and three optical counterparts, which create a multiple core system, is reported by Kale et al. (2013).

Two other radio sources are visible in the field to the north (co-located with an optical source with unknown redshift) and to the south-west (likely connected to a clustermember galaxy). The $\mathrm{X}$-ray morphology is elongated in 

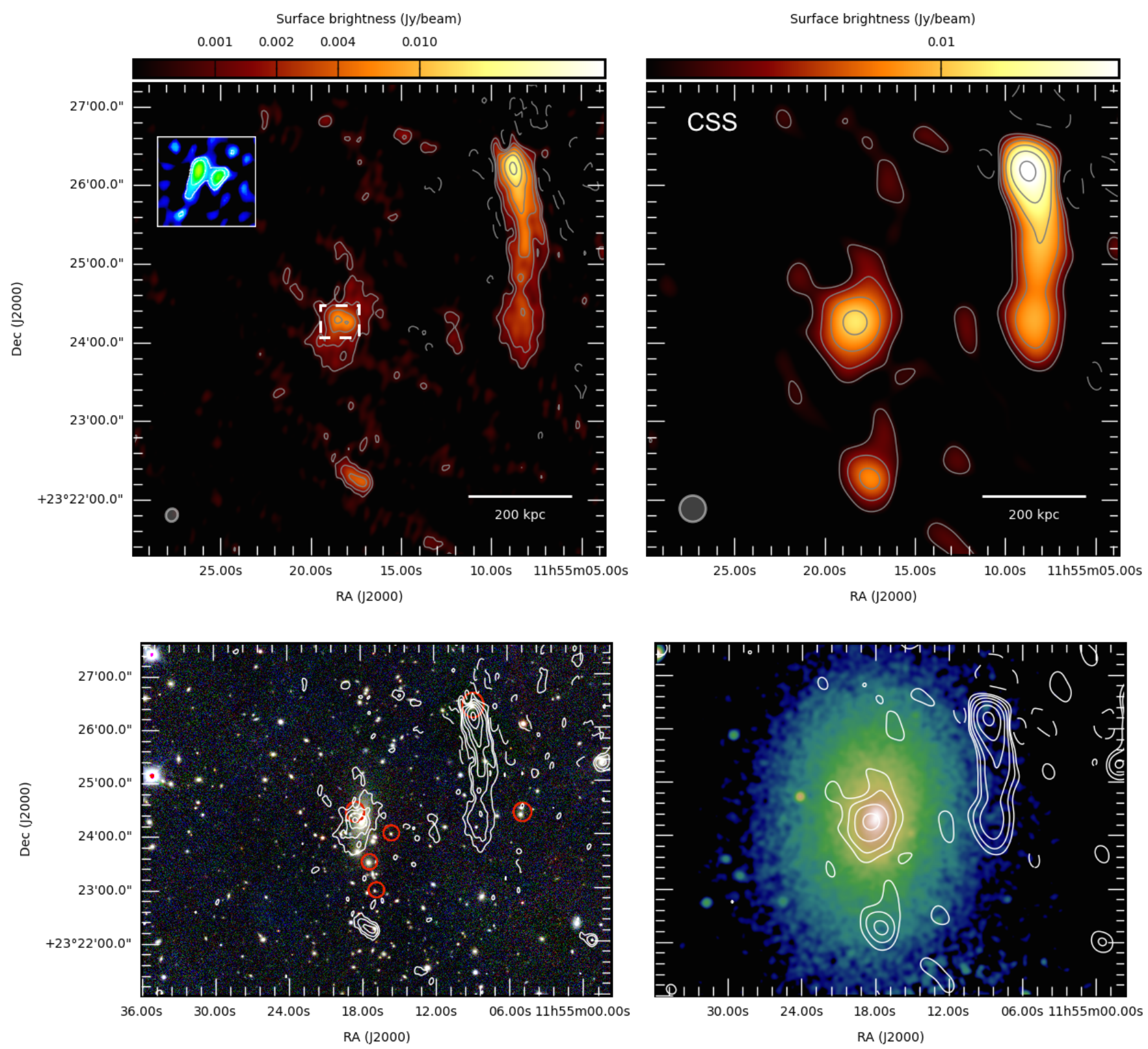

Fig. 6. A1413. Top left panel: high-resolution $144 \mathrm{MHz}$ LOFAR image of A1413. The contour levels start at $3 \sigma$, where $\sigma=270 \mu \mathrm{Jy}$ beam ${ }^{-1}$, and are spaced by a factor of two. The negative contour level at $-3 \sigma$ is overlaid with a dashed line. The beam is $10^{\prime \prime} \times 9^{\prime \prime}$ and is shown in grey in the bottom left corner of the image. The insert box shows the $u v$-cut high-resolution image of the central sources that are subtracted to obtained the image in top right panel. Top right panel: low-resolution $144 \mathrm{MHz}$ LOFAR image of A1413. The contour levels start at $3 \sigma$, where $\sigma=450 \mu \mathrm{Jy}$ beam ${ }^{-1}$, and are spaced by a factor of two. The negative contour level at $-3 \sigma$ is overlaid with a dashed line. The beam is $20^{\prime \prime} \times 20^{\prime \prime}$ and is shown in grey in the bottom left corner of the image. This image was obtained after the CSS with a taper of $20^{\prime \prime}$ and Briggs weighting (robust $=0$ ). Bottom left panel: optical SDSS image of A1413 with the high-resolution $\left(10^{\prime \prime} \times 9^{\prime \prime}\right) 144 \mathrm{MHz}$ LOFAR contours overlaid. The red circles indicate the cluster-member galaxies with available spectroscopic redshift. Bottom right panel: Chandra X-ray image of A1413 smoothed on a scale of 5" with the low-resolution $\left(20^{\prime \prime} \times 20^{\prime \prime}\right) 144 \mathrm{MHz}$ LOFAR contours overlaid.

the east-west direction and does not show a strong central peak. Moreover, based on weak lensing analysis, Dahle et al. (2002) inferred significant dynamical activity. An upper limit on the radio power was derived by Venturi et al. (2008) using a GMRT observation at $610 \mathrm{MHz}$. The upper limit rescaled at $1.4 \mathrm{GHz}$ is $P_{1.4} \leq 0.64 \times 10^{24} \mathrm{~W} \mathrm{~Hz}^{-1}$ (Cassano et al. 2013).

During the LOFAR observation of this cluster, the ionosphere was very active and we reached a noise of $500 \mu \mathrm{Jy}_{\text {beam }}{ }^{-1}$ (Fig. 8). At this sensitivity level, no radio diffuse emission is detected at $144 \mathrm{MHz}$ either after subtracting the contribution of the compact sources. Hence, we compute a new upper limit on the radio power by injecting a mock halo in the data set (see Sect. 4). The integrated flux density of the mock halo computed within a region centred on the injected halo with a radius of $R_{\mathrm{H}}=$ $429 \mathrm{kpc}$ is $37 \mathrm{mJy}$ at $144 \mathrm{MHz}$, which corresponds to a total radio power at $144 \mathrm{MHz}$ of $P_{144}=11.9 \times 10^{24} \mathrm{~W} \mathrm{~Hz}^{-1}$. Assuming the typical spectral index value used in the power-mass correlation for haloes $(\alpha=-1.3)$, we derived the radio power that we plot in Fig. 14. The new upper limit on the radio power at $1.4 \mathrm{GHz}$ is $P_{1.4}<0.62 \times 10^{24} \mathrm{~W} \mathrm{~Hz}^{-1}$, i.e. comparable with that derived in literature. 

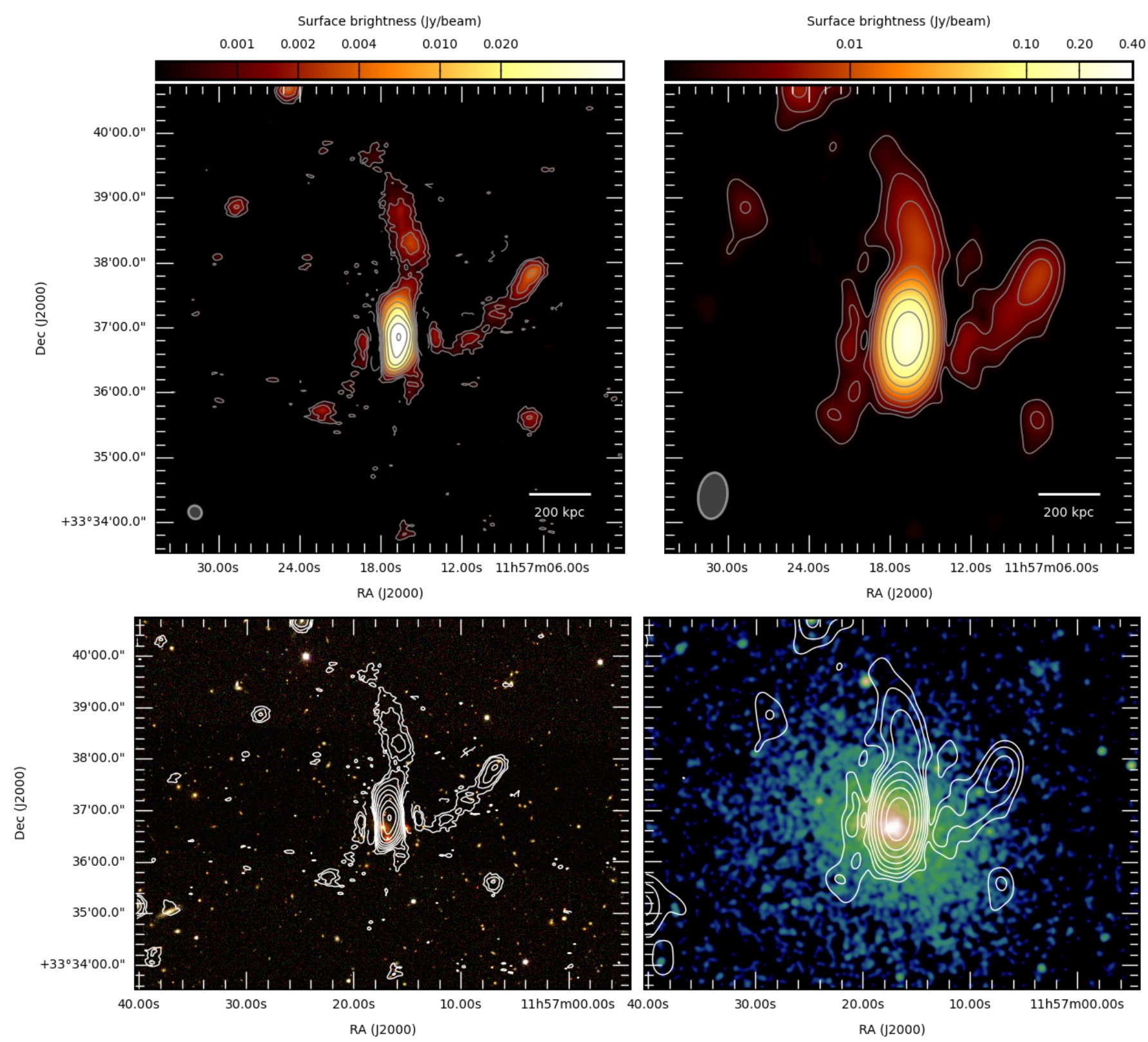

Fig. 7. A1423. Top left panel: high-resolution $144 \mathrm{MHz}$ LOFAR image of A1423. The contour levels start at $3 \sigma$, where $\sigma=170 \mu \mathrm{Jy}$ beam ${ }^{-1}$, and are spaced by a factor of two. The negative contour level at $-3 \sigma$ is overlaid with a dashed line. The beam is $13^{\prime \prime} \times 12^{\prime \prime}$ and is shown in grey in the bottom left corner of the image. Top right panel: low-resolution $144 \mathrm{MHz}$ LOFAR image of A1423. The contour levels start at $3 \sigma$, where $\sigma=420 \mu \mathrm{Jy} \mathrm{beam}^{-1}$, and are spaced by a factor of two. The negative contour level at $-3 \sigma$ is overlaid with a dashed line. The beam is $43^{\prime \prime} \times 27^{\prime \prime}$ and is shown in grey in the bottom left corner of the image. Bottom left panel: optical Pan-STARRS image of A1423 with the high-resolution $\left(43^{\prime \prime} \times 27^{\prime \prime}\right) 144 \mathrm{MHz}$ LOFAR contours overlaid. The red circles indicate the cluster-member galaxies with available spectroscopic redshift. Bottom right panel: Chandra X-ray image of A1423 smoothed on a scale of 5" with the low-resolution (43" $\left.\times 27^{\prime \prime}\right) 144$ MHz LOFAR contours overlaid.

\section{7. $R X J 1720.1+2638$}

The cool-core cluster RXJ1720.1+2638 has been studied in detail by Giacintucci et al. (2014a) using VLA and GMRT observations. The central source is classified as a mini halo consisting of a bright central component with a size of $\sim 160 \mathrm{kpc}$, and a fainter spiral-shaped tail of emission extending towards the south for more than $200 \mathrm{kpc}$. Two cold fronts detected in the Chandra $\mathrm{X}$-ray image of the cluster appear to confine the mini halo.

The LOFAR images, shown in Fig. 9, reveal a new diffuse component extending beyond the cold fronts, not visible at higher frequencies. The emission extends towards the south-west with an overall size of $\sim 600 \mathrm{kpc}$. We reprocessed the GMRT observation at $610 \mathrm{MHz}$ and obtained the high-resolution image shown in the left panel of Fig. 10. The head-tail radio galaxy to the northeast of the cluster is clearly visible and a connection between the central diffuse emission and the tail is already seen at this frequency. We re-imaged the LOFAR and GMRT data sets with a Gaussian taper of $20^{\prime \prime}$ and with the same pixel size, baseline range (200-40000 $\lambda$ ), and uniform weighting scheme to minimise the differences in the $u v$-coverage of the two observations and obtained a spectral index map that is shown in the right panel of Fig. 10. The mini halo appears to have a constant spectrum with a spectral index of $\alpha_{144}^{610} \sim-1$, whilst the head-tail radio 

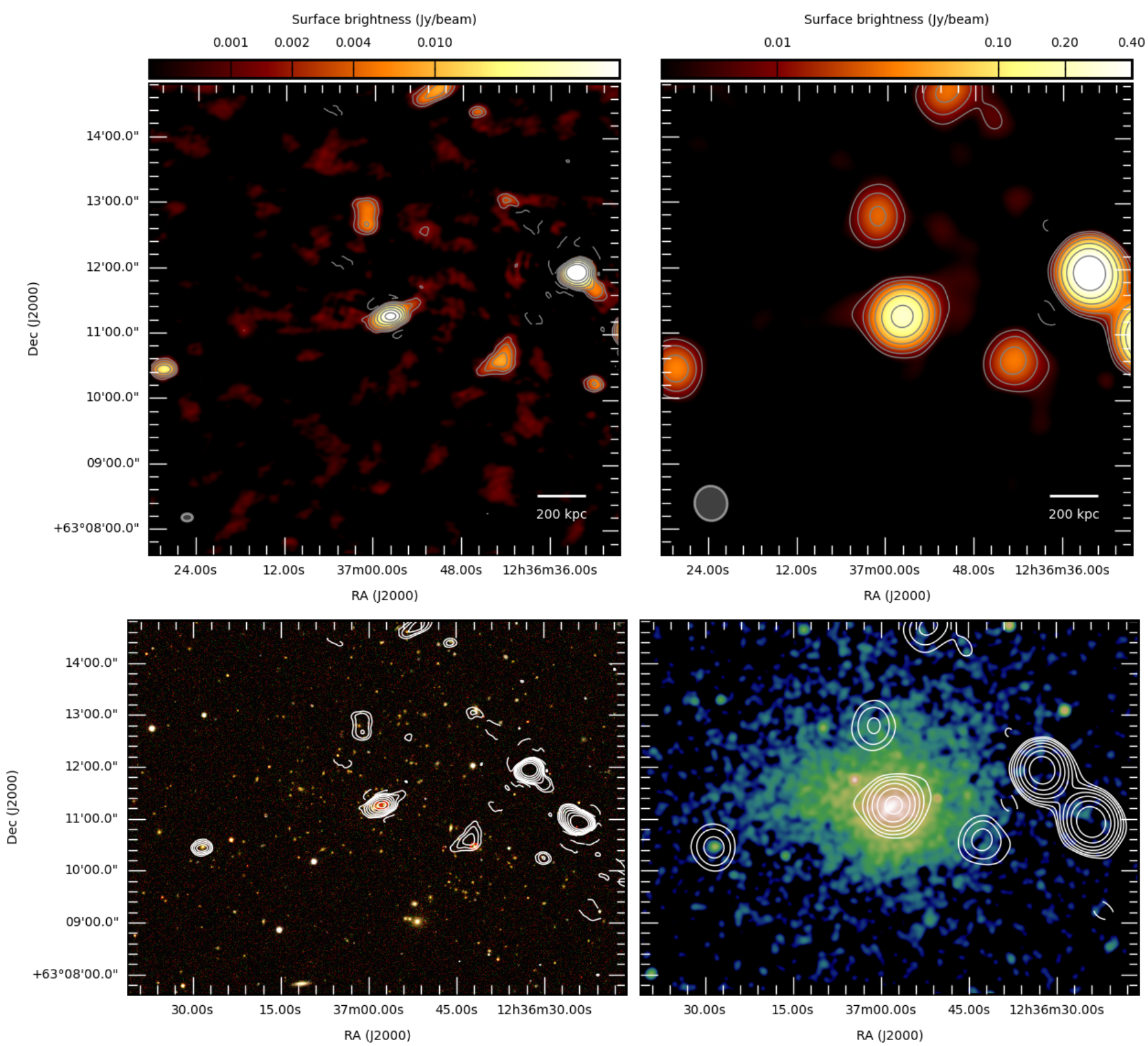

Fig. 8. A1576. Top left panel: high-resolution $144 \mathrm{MHz}$ LOFAR image of A1576. The contour levels start at $3 \sigma$, where $\sigma=500 \mu \mathrm{Jy} \mathrm{beam}{ }^{-1}$, and are spaced by a factor of two. The negative contour level at $-3 \sigma$ is overlaid with a dashed line. The beam is $10^{\prime \prime} \times 7^{\prime \prime}$ and is shown in grey in the bottom left corner of the image. Top right panel: low-resolution $144 \mathrm{MHz}$ LOFAR image of A1576. The contour levels start at $3 \sigma$, where $\sigma=150$ $\sigma=2 \mathrm{mJy}_{\text {beam }}^{-1}$, and are spaced by a factor of two. The negative contour level at $-3 \sigma$ is overlaid with a dashed line. The beam is $32^{\prime \prime} \times 30^{\prime \prime}$ and is shown in grey in the bottom left corner of the image. Bottom left panel: optical Pan-STARRS image of A1576 with the high-resolution $\left(10^{\prime \prime} \times 7^{\prime \prime}\right) 144 \mathrm{MHz}$ LOFAR contours overlaid. The red circles indicate the cluster-member galaxies with available spectroscopic redshift. Bottom right panel: Chandra X-ray image of A1576 smoothed on a scale of 5" with the low-resolution (32" $\left.\times 30^{\prime \prime}\right) 144$ MHz LOFAR contours overlaid.

galaxy has the typical trend of an active radio galaxy with a flat core and a steepening along the tail. The emission connecting the tail and the mini halo is steep, ranging between $\alpha_{144}^{610} \sim-1.4$ and $\alpha_{144}^{610} \sim-1.8$. The cluster-scale diffuse emission, which cannot be seen at $610 \mathrm{MHz}$, is ultra steep, and we can only provide upper limits, as shown in Fig. 10, using the mean flux density from the LOFAR image and the rms noise from the GMRT image. The radio emission in RXJ1720.1+2638 resembles that of PSZ1G139.61+24 (Savini et al. 2018a) with an inner, flatter component in the form of the already-known mini halo, and an outer part with a steeper-spectrum halo-like emission on larger scales, with $\alpha_{144}^{610}<-1.5$. The two inner cold fronts (reported in the X-ray image of Fig. 9) appear to separate the two components. Interestingly, the cluster A2142 (Venturi et al. 2017) also shows a two-component emission, and the presence of cold fronts both in the inner and outer region. However, because of the scarcity of X-ray counts in the outer cluster regions, we are not able to search for cold fronts on a larger scale.

\section{8. $A 2261$}

A2261 is a non-cool-core cluster with a central radio source coincident with the BCG. The cluster has a relaxed morphology in Xrays in the central $500 \mathrm{kpc}$-radius region, while showing a diffuse 

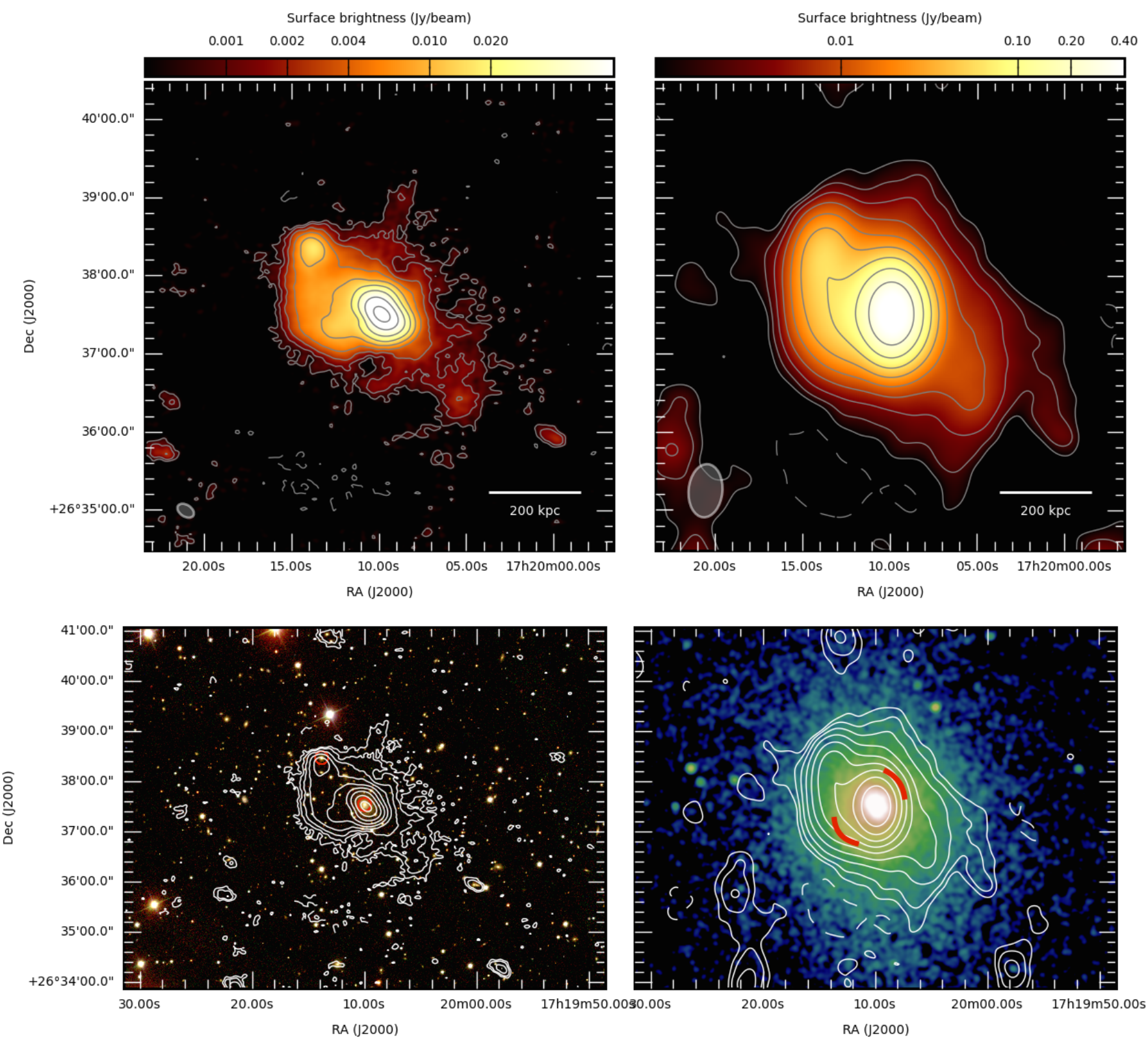

Fig. 9. RXJ1720.1+2638. Top left panel: high-resolution $144 \mathrm{MHz}$ LOFAR image of RXJ1720.1+2638. The contour levels start at $3 \sigma$, where $\sigma=200 \mu \mathrm{Jy}_{\text {beam }}{ }^{-1}$, and are spaced by a factor of two. The negative contour level at $-3 \sigma$ is overlaid with a dashed line. The beam is $14^{\prime \prime} \times 9^{\prime \prime}$ and is shown in grey in the bottom left corner of the image. Top right panel: low-resolution $144 \mathrm{MHz}$ LOFAR image of RXJ1720.1+2638. The contour levels start at $3 \sigma$, where $\sigma=330 \mu \mathrm{Jy} \mathrm{beam}^{-1}$, and are spaced by a factor of two. The negative contour level at $-3 \sigma$ is overlaid with a dashed line. The beam is $41^{\prime \prime} \times 26^{\prime \prime}$ and is shown in grey in the bottom left corner of the image. Bottom left panel: optical Pan-STARRS image of RXJ1720.1+2638 with the high-resolution $\left(14^{\prime \prime} \times 9^{\prime \prime}\right) 144 \mathrm{MHz}$ LOFAR contours overlaid. The red circles indicate the cluster-member galaxies with available spectroscopic redshift. Bottom right panel: Chandra X-ray image of RXJ1720.1+2638 smoothed on a scale of 5" with the lowresolution $\left(41^{\prime \prime} \times 26^{\prime \prime}\right) 144 \mathrm{MHz}$ LOFAR contours overlaid. The red arcs indicate the position of the two cold fronts found by Giacintucci et al. (2014a).

patch of X-ray emission towards the west of the cluster; this is likely an infalling group, which suggests a possible minor merger (von der Linden et al. 2014). Sommer et al. (2017) found a centrally located diffuse component on a scale of $\sim 1 \mathrm{Mpc}$ using VLA observations at $1.4 \mathrm{GHz}$ and classified the large-scale source as a radio halo.

The high-resolution LOFAR image (left panel of Fig. 11) shows the presence of a central compact radio source and a radio galaxy located at $\sim 540 \mathrm{kpc}$ towards the north-west of the cluster centre; this radio galaxy has a faint, diffuse emission, symmetric with respect to its core, which could be the two remnant radio lobes. The core is identified with an X-ray source and an optical galaxy whose redshift is unknown. At the cluster redshift, the radio galaxy would extend up to $\sim 590 \mathrm{kpc}$. A patch of diffuse radio emission with no clear origin, which might be a relic or a dying AGN, is seen to the west of the cluster centre. After modelling these compact sources using only the longer baselines with a $u v$-cut of $>750 \lambda$ (that corresponds to $1 \mathrm{Mpc}$ ), we subtracted their contribution, revealing the presence of diffuse emission extending up to $1.2 \mathrm{Mpc}$. In general, the $u v$-subtraction method is not indicated for extended sources and in this case the remaining contribution of the diffuse lobes of the radio galaxy 

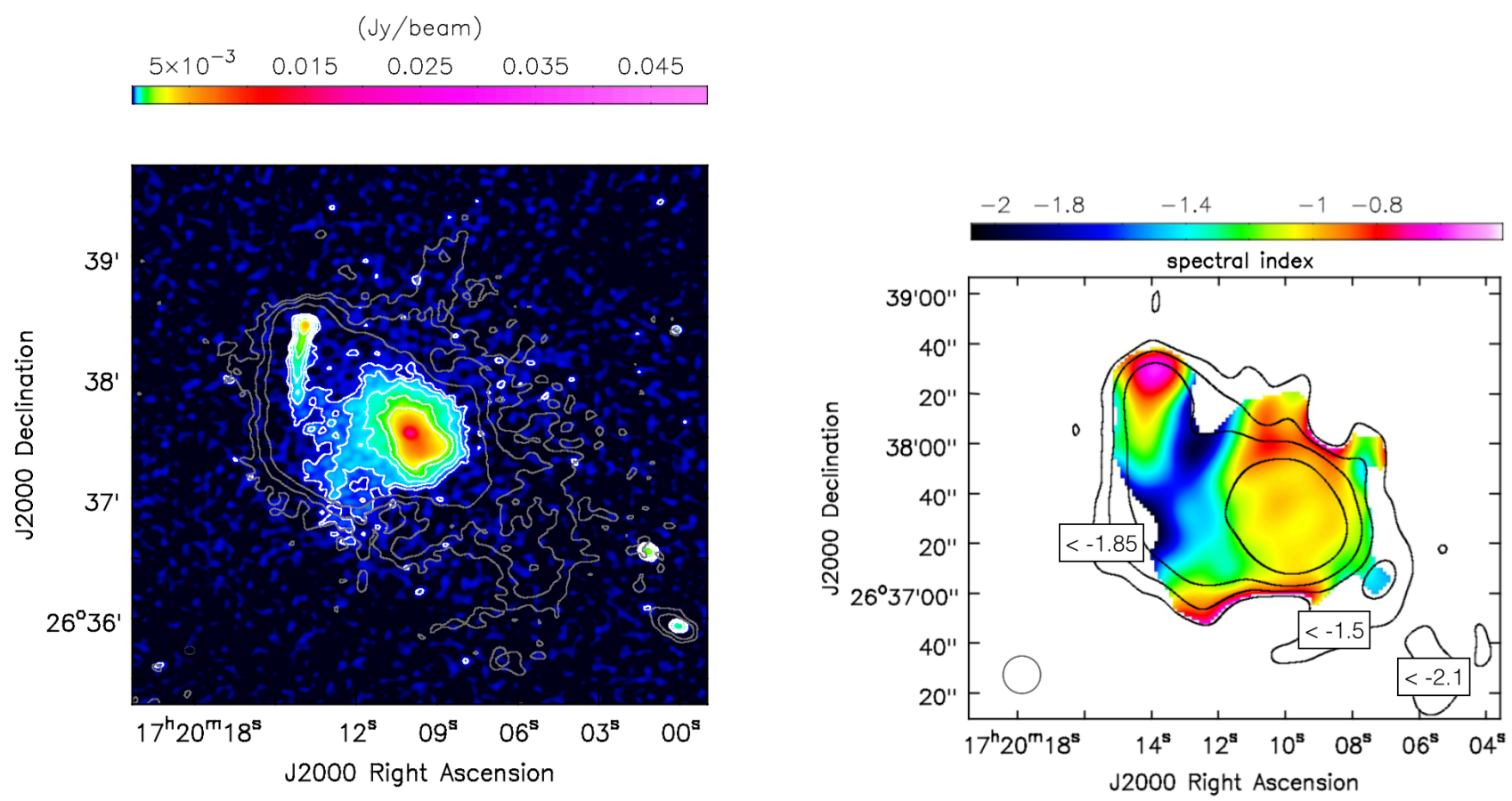

Fig. 10. Left panel: $610 \mathrm{MHz}$ GMRT image of RXJ1720.1+2638 with its contour levels in white starting at $3 \sigma$, where $\sigma=45 \mu \mathrm{Jy}_{\text {beam }}{ }^{-1}$ spaced by a factor of two. The first three contour levels from the high-resolution LOFAR image of Fig. 9 are overlaid in grey. The beam are $5^{\prime \prime} \times 5^{\prime \prime}$ and $14^{\prime \prime} \times 9^{\prime \prime}$ for GMRT and LOFAR, respectively, and are shown in the bottom left corner of the image. Right panel: spectral index map between the $610 \mathrm{MHz}$ GMRT and $144 \mathrm{MHz}$ LOFAR images of RXJ1720.1+2638. The contour levels, overlaid in black, are from the LOFAR image obtained for the spectral analysis (uniform weighting, $u v$-cut, see main text), not shown in the paper. Pixels below $3 \sigma$ are blanked. The error map is shown in Fig. A.3.

can be clearly seen. This affects the morphology of the diffuse emission that shows the brightness peak and an extension at the location of the radio galaxy and the unknown patch of diffuse emission. We note that the image obtained by Sommer et al. (2017; Fig. 2) shows a different morphology of the large-scale diffuse source, which is likely due to the result of the $u v$ subtraction at a different frequency. For instance, the fact that the radio galaxy lobes are better subtracted might be related to their probable steep spectrum that contributes at high frequencies to a much smaller extent compared to what is observed at low frequencies. However, the radio source as seen at $144 \mathrm{MHz}$ extends on a larger scale than the single radio sources, around the cluster centre, and also towards west and south-west, where the patch of X-ray emission is located, which might indicate a minor merger. This could also explain the offset between radio and X-ray emission peaks, also visible in the $1.4 \mathrm{GHz}$ image. We obtained images using different $u v$-cut for subtraction corresponding to $800 \mathrm{kpc}, 1 \mathrm{Mpc}$, and 1.2 Mpc. We show in Fig. A.5, models, and resulting images. Although the $u v$-subtraction is not entirely reliable as for the case of a compact point source, we can state that diffuse emission that is not related to the radio galaxy is clearly visible. Hence, the LOFAR image at $144 \mathrm{MHz}$ confirms the presence of a radio halo, as suggested by the VLA observation at $1.4 \mathrm{GHz}$, although its morphology and radio power are hard to determine. We estimate the contribution of the flux densities of the radio galaxy and unknown patch of emission, re-imaging the LOFAR $u v$-subtracted data set with the same taper and weighting scheme used for the halo (Fig. 11 in the paper), keeping, in addition, the $u v$-cut of $750 \lambda$ used for modelling the single sources. Subtracting this value of $20 \mathrm{mJy}$ from the total flux density of the large-scale diffuse emission that is $\sim 185 \mathrm{mJy}$; the radio halo has $\sim 165 \mathrm{mJy}$ corresponding to a total radio power at $144 \mathrm{MHz}$ of $\sim 26 \times 10^{24} \mathrm{~W} \mathrm{~Hz}^{-1}$.
Considering the total flux density at $240 \mathrm{MHz}, 610 \mathrm{MHz}$, and 1.4 GHz reported in Table 3 in Sommer et al. (2017), we can provide an approximate estimate of the spectral index of the halo including LOFAR measurements to be $\alpha=-1.7 \pm 0.3$, which makes the radio halo in A2261 a candidate USS halo.

\section{9. $A 2390$}

The cool-core cluster A2390 is particularly interesting for its large mass $\left(\sim 10^{15} M_{\odot}\right)$, which sets it apart from the other clusters in the sample (see top left panel of Fig. 15). A2390 has been observed at $1.4 \mathrm{GHz}$ with the VLA. The data were analysed by Bacchi et al. (2003), who classified the detected emission as a mini halo with an integrated flux density of $63 \pm 3 \mathrm{mJy}$ and an extension of $\sim 550 \mathrm{kpc}$. Using deeper Jansky VLA observations in the $1-2 \mathrm{GHz}$ frequency band and re-analysing the VLA observation in Bacchi et al. (2003), Sommer et al. (2017) discovered diffuse radio emission on a larger scale extending for $\sim 800 \mathrm{kpc}$ in the form of a radio halo, after subtracting the compact source contribution. A spectral in-band analysis was also performed, finding a steep spectrum of $\alpha_{2 \mathrm{GHz}}^{1 \mathrm{GHz}}=-1.60 \pm 0.17$.

Our high-resolution LOFAR image shows that the central radio source is a double radio galaxy with the lobes extending in the east-west axis for $\sim 600 \mathrm{kpc}$. The morphology indicates the presence of a bright core and fading lobes. Radio galaxies of such a size are not common at the centre of clusters, where the ICM usually prevents the expansion of the lobes to such large scales. The absence of emission in the north-south axis around the galaxy core had already been pointed out in previous works by Bacchi et al. (2003) and Sommer et al. (2017). We note that there are some imaging artefacts due to the imperfect calibration of the galactic core that is brighter than the diffuse lobes and causes the image sensitivity to be limited by the dynamic range. The 

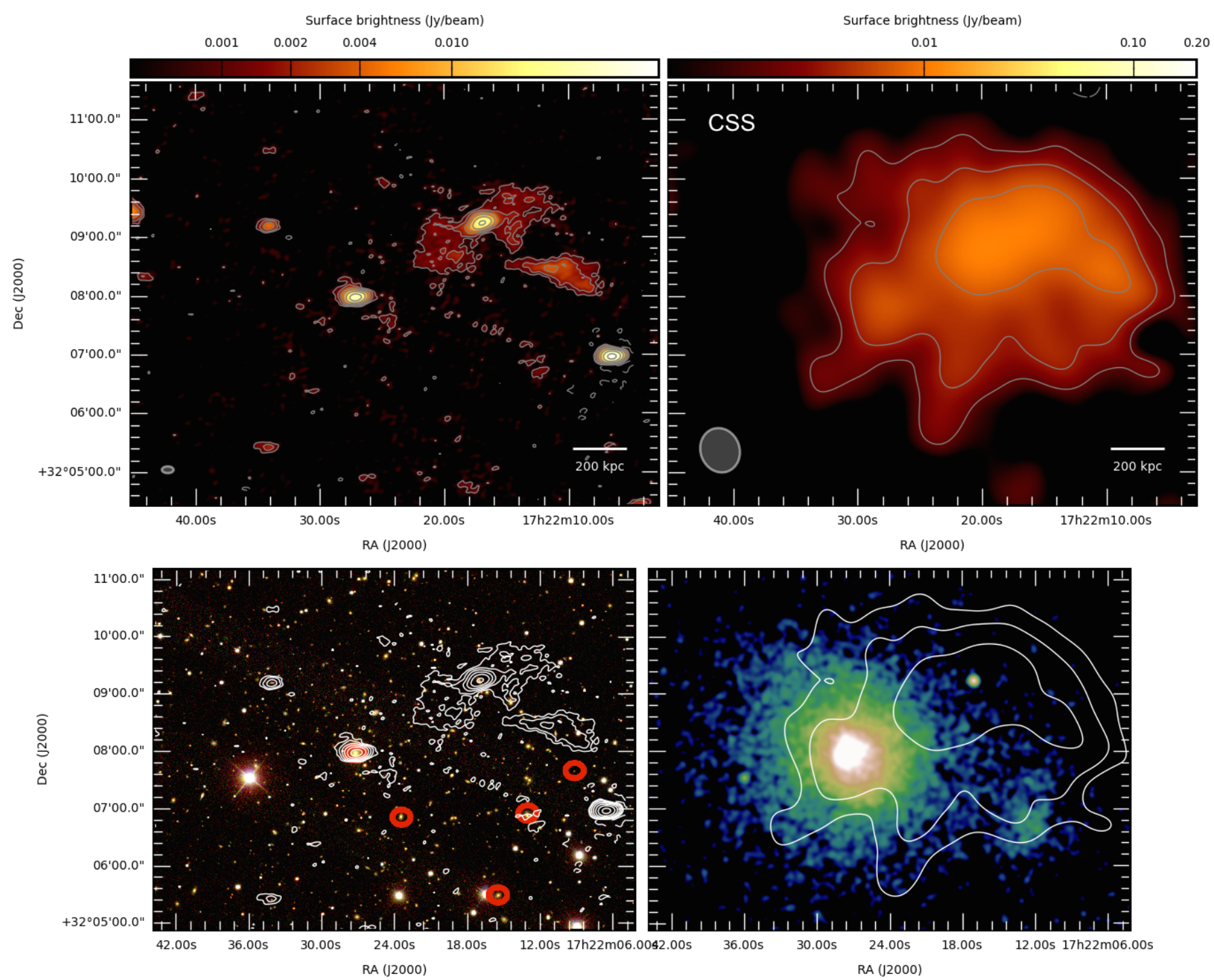

Fig. 11. A2261. Top left panel: high-resolution $144 \mathrm{MHz}$ LOFAR image of A2261. The contour levels start at $3 \sigma$, where $\sigma=280 \mu \mathrm{Jy}$ beam ${ }^{-1}$, and are spaced by a factor of two. The negative contour level at $-3 \sigma$ is overlaid with a dashed line. The beam is $12^{\prime \prime} \times 7^{\prime \prime}$ and is shown in grey in the bottom left corner of the image. Top right panel: low-resolution $144 \mathrm{MHz}$ LOFAR image of A2261. The contour levels start at $3 \sigma$, where $\sigma=600 \mu \mathrm{Jy}_{\text {beam }}{ }^{-1}$, and are spaced by a factor of two. The negative contour level at $-3 \sigma$ is overlaid with a dashed line. The beam is $46^{\prime \prime} \times 40^{\prime \prime}$ and is shown in grey in the bottom left corner of the image. This image was obtained after CSS with a taper of $35^{\prime \prime}$ and Briggs weighting (robust $=0$ ). Bottom left panel: optical SDSS image of A2261 with the high-resolution $\left(12^{\prime \prime} \times 7^{\prime \prime}\right) 144 \mathrm{MHz}$ LOFAR contours overlaid. The red circles indicate the cluster-member galaxies with available spectroscopic redshift. Bottom right panel: Chandra X-ray image of A2261 smoothed on a scale of $5^{\prime \prime}$ with the low-resolution $\left(46^{\prime \prime} \times 40^{\prime \prime}\right) 144 \mathrm{MHz}$ LOFAR contours overlaid.

low-resolution LOFAR image shows the presence of diffuse emission on a larger scale with a total extension of $1.2 \mathrm{Mpc}$. The lowest contour of the low-resolution image might still be interpreted as cluster-scale diffuse emission or old lobes emission. It is not possible to disentangle this emission from that of the fading lobes of the radio galaxy, neither it is possible to accurately subtract its core to search for the presence of an underlying radio halo. Hence, we can only conclude that the radio emission is mostly dominated by the contribution of the radio galaxy whose morphology is clearly visible with LOFAR.

In the left panel of Fig. 13, we show the image of the VLA data set that was calibrated in Sommer et al. (2017). We reimaged this data set to match ${ }^{6}$ the LOFAR imaging parameters,

\footnotetext{
6 In this case we did not apply a $u v$-cut and the uniform weighting parameter (as we did for the spectral analysis of A2261) because this would prevent us from recovering the morphology of the diffuse lobes.
}

such as cell size and resolution, and obtain the spectral index map shown in the right panel of Fig. 13. The core appears to have a flat spectrum with $\alpha_{144}^{1400} \sim-0.5$, which is a typical value for the core of an active radio galaxy, whilst the lobes are much steeper with $\alpha_{144}^{1400}$ ranging between $\sim-1.3$ and $\sim-2$. We can speculate on the type and evolutionary phase of the radio galaxy. It could be an FR-II radio galaxy that has recently restarted (which would explain the flat core), still showing old lobes and relic hotspots from a previous activity cycle (e.g. Shulevski et al. 2015, Brienza et al. 2016). Interestingly, Augusto et al. (2006) classified the radio galaxy of A2390 as an FR-II with a flat-spectrum core and a compact twin-jet structure in a north-south direction on a sub-arcsec scale, as seen in the $1.7-43 \mathrm{GHz}$ frequency

However, we note that the shortest baselines of the VLA and LOFAR observations are able to detect large-scale emission up to few arcminutes, larger than the emission in A2390. 

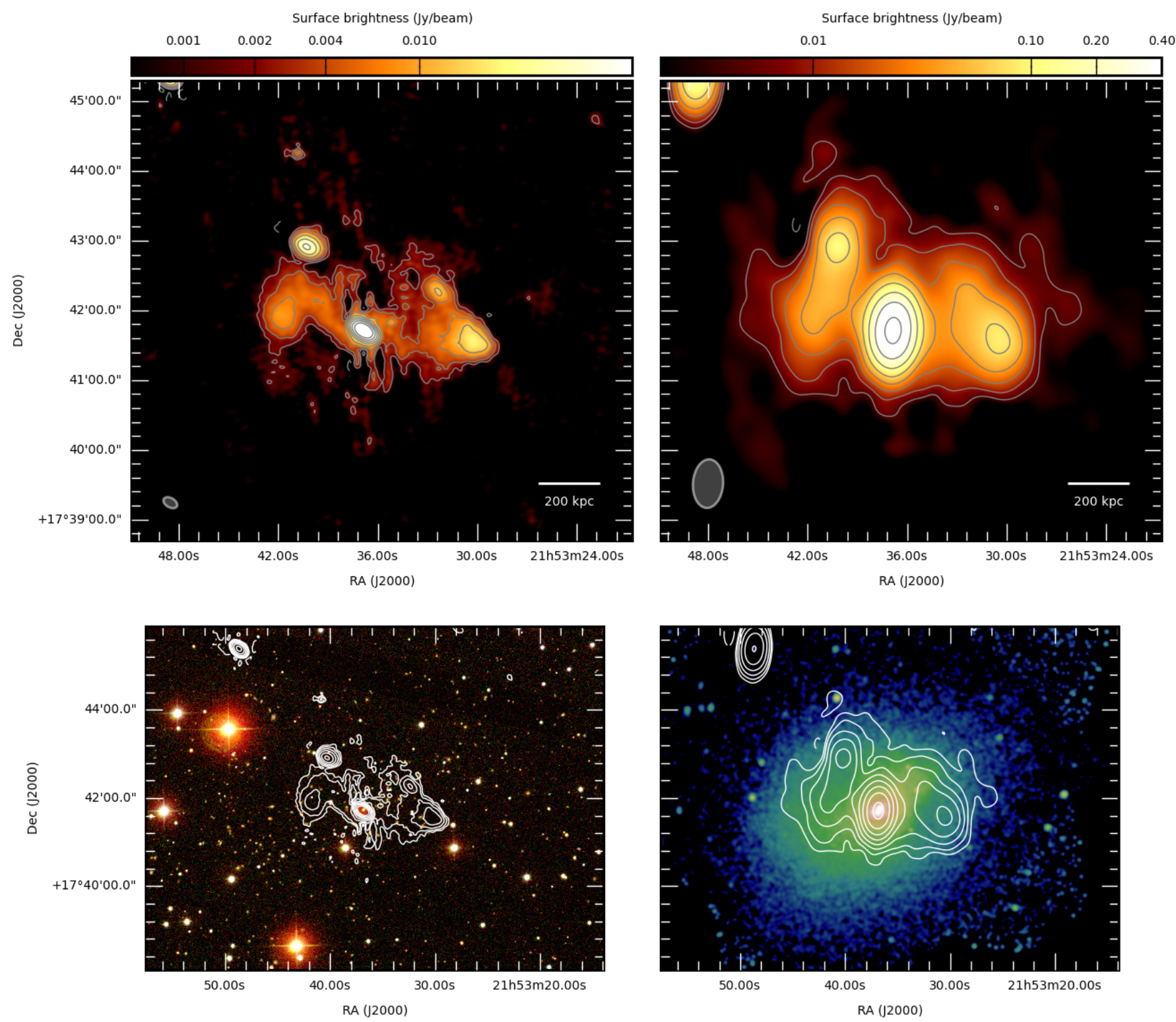

Fig. 12. A2390. Top left panel: high-resolution $144 \mathrm{MHz}$ LOFAR image of A2390. The contour levels start at $3 \sigma$, where $\sigma=400 \mu \mathrm{Jy}$ beam ${ }^{-1}$, and are spaced by a factor of two. The negative contour level at $-3 \sigma$ is overlaid with a dashed line. The beam is $13^{\prime \prime} \times 8^{\prime \prime}$ and is shown in grey in the bottom left corner of the image. Top right panel: low-resolution $144 \mathrm{MHz}$ LOFAR image of A2390. The contour levels start at $3 \sigma$, where $\sigma=1.2 \mathrm{mJy}_{\text {beam }}{ }^{-1}$, and are spaced by a factor of two. The negative contour level at $-3 \sigma$ is overlaid with a dashed line. The beam is $42^{\prime \prime} \times 26^{\prime \prime}$ and is shown in grey in the bottom left corner of the image. Bottom left panel: optical Pan-STARRS image of A2390 with the highresolution $\left(13^{\prime \prime} \times 8^{\prime \prime}\right) 144 \mathrm{MHz}$ LOFAR contours overlaid. Bottom right panel: Chandra X-ray image of A2390 smoothed on a scale of 5" with the low-resolution $\left(42^{\prime \prime} \times 26^{\prime \prime}\right) 144 \mathrm{MHz}$ LOFAR contours overlaid.

range with very long baseline interferometry (VLBI) observations. They also note that the orientation of the jets is misaligned with respect to the ionisation cones and dust disc of the host galaxy on larger scales. They suggest that the misalignment might be due to a precession of the central super massive black hole, and that the radio source might be an example of a bubble being blown into the ICM at its early stage $\left(10^{3}-10^{4} \mathrm{yr}\right.$ duration). This is in line with our interpretation that the east-west jets are originated by a previous AGN active phase. The AGN might then be experiencing a second episode of activity with the jets growing in the north-south direction after a precession. With this scenario we expect the presence of X-ray cavities and bubbles. Four inner cavities are found by Sonkamble et al. (2015) and coincide with the location of the inner jets (east-west and north-south). Further cavities on large scales at the location of the old lobes are also expected and could be searched in future studies.

\section{Discussion}

On the basis of the X-ray morphology, the nine clusters presented in this work are not currently undergoing a major merger. As shown in the top panels of Fig. 15, five of these clusters host a cool core, whilst the remaining host a warm core, according to the classification based on the central gas entropy value (Giacintucci et al. 2017). The overall picture as seen in the lowfrequency radio band by LOFAR is very diverse with the presence of radio diffuse emission in the form of two radio haloes, three mini haloes, and two uncertain cases, while two clusters do not host diffuse emission at all. Even though the sample we 

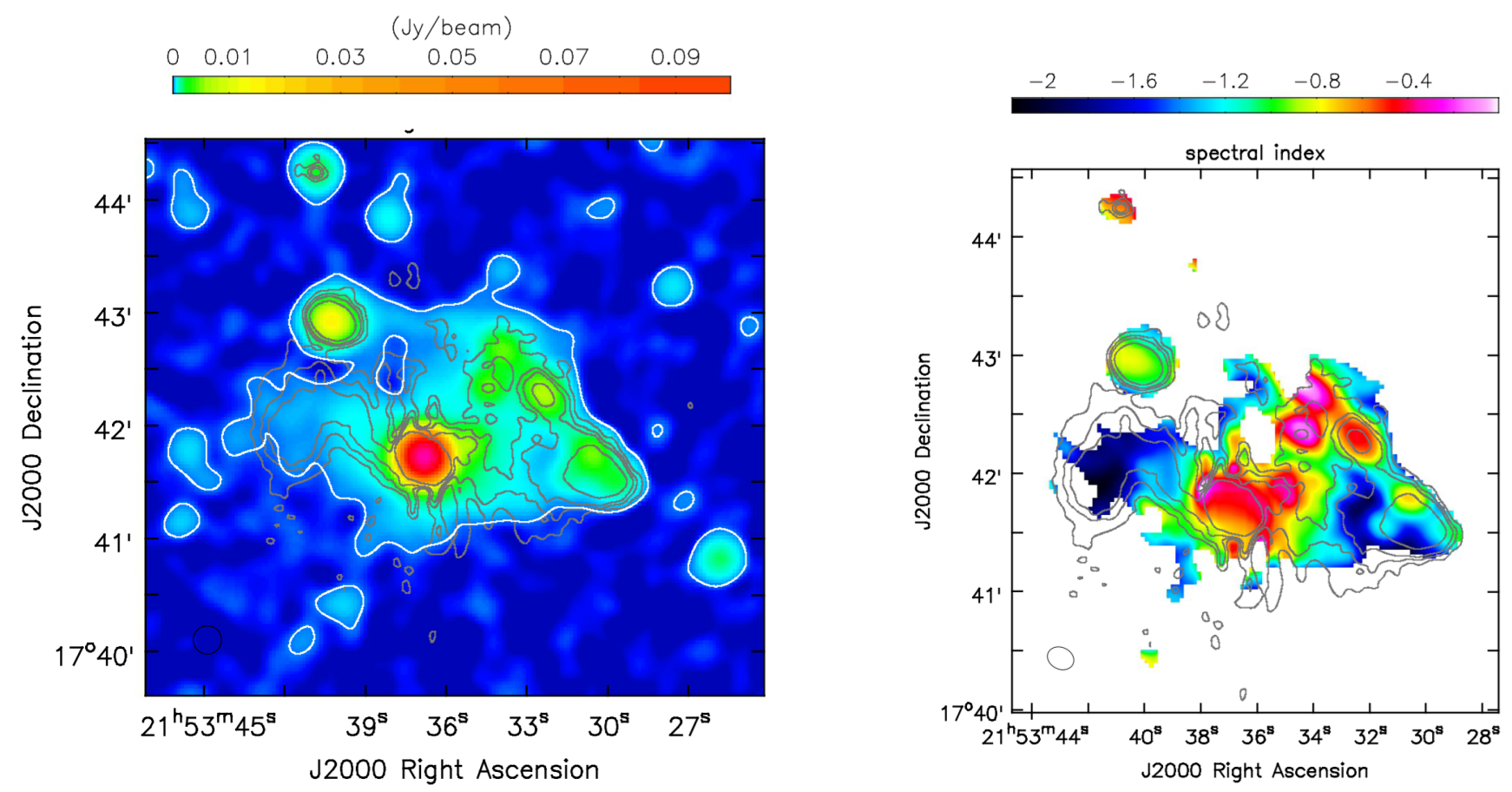

Fig. 13. Left panel: $1.5 \mathrm{GHz}$ VLA image of A2390 with a beam of $15^{\prime \prime} \times 15^{\prime \prime}$ and an rms noise of $26 \mu \mathrm{Jy} \mathrm{beam}^{-1}$. The first $3 \sigma$ contour is shown in white. Right panel: spectral index map between the $1.5 \mathrm{GHz}$ VLA and $144 \mathrm{MHz}$ LOFAR images of A2390. The contour levels, overlaid in grey, are from the LOFAR high-resolution image in Fig. 12 with $(4,8,12,24) \times \sigma$ where $\sigma=400 \mu \mathrm{Jy} \mathrm{beam}^{-1}$. Pixels below $3 \sigma$ are blanked. The error map is shown in Fig. A.4.

studied is not large enough to derive statistical results, we note that this is the largest sample of galaxy clusters studied within LoTSS, and we can draw a number of conclusions that can also be indicative for future low-frequency observations. In our sample, the diffuse radio emission appears to be uncorrelated with the dynamical state as indicated by the centroid shifts, $w$, of the $\mathrm{X}$-ray emission computed at $500 \mathrm{kpc}$. Even looking at $w$ on smaller scales, from 200 to $500 \mathrm{kpc}$, as shown in the bottom left panel of Fig. 15, no correlation between the radio emission and $w$ is found. Clusters that possess similar dynamical properties do not show the same radio properties, for example the non-coolcore clusters RXC0142.0+2131 and A1576, which have similar $\mathrm{X}$-ray properties (relaxed morphology, comparable $c$ and $K_{0}$ ) and also comparable cluster masses, host a radio halo and no diffuse emission, respectively. Also plotting the power at $144 \mathrm{MHz}$ versus the ratio of the X-ray concentration parameter, $c$, to $w$ (see bottom right panel of Fig. 15) does not reveal a clear connection between the radio emission and the dynamical state of the cluster. Haloes are not necessary found in clusters with low $c$ and high $w$; see for example A2261.

In the top right panel of Fig. 15, we plot $c$ versus $K_{0}$ for each cluster. Two-component radio haloes (MH+USSH) are found in clusters with high $c$ and low $K_{0}$, while giant haloes are detected in clusters with low $c$ and higher $K_{0}$. Clusters for which no radio emission is found at the sensitivity level of these observations are found in both sides of the plot. Finally, a mini halo, which is the only mini halo found in a non-cool-core cluster that is also in the sample studied in Giacintucci et al. (2017), is found in a cluster with low $c$ and high $K_{0}$.

Our observations show that the two clusters, PSZ1G139.61+24 and RXJ1720.1+2638, that host a mini halo and have the largest value of $c$, also show larger-scale ultra-steep diffuse emission that extends beyond the cold fronts. In our sample, the only mini halo that is confined to the central region of a cluster is hosted by A1413, which is not a cool-core cluster. This might indicate that the presence of a dense cool core is required to initiate the re-acceleration of CRe in the regions surrounding the core through gas sloshing triggered by a minor merger, as suggested for PSZ1G139.61+24 by Savini et al. (2018a). The observation that mini haloes can have a flatter core that is surrounded by a faint, steep corona is helpful in resolving their origin. A contribution from hadronic collisions cannot be excluded for the central region of cool-core clusters, but this contribution cannot explain the large-scale ultra-steep emission (e.g. Brunetti et al. 2008, Macario et al. 2010). Hence, different scenarios for the cosmic-ray origin might co-exist in these clusters that show hints of gas sloshing, with a distinction between the region within and outside the core.

In our sample, no giant radio haloes in cool-core clusters are found. A2261 and RXC0142.0+2131, where haloes are found, do not host a cool core and the extended emission in A2390 is likely to merely originate from the central radio galaxy, whose very diffuse lobes could have accidentally been classified as a halo at $1.4 \mathrm{GHz}$ in Sommer et al. (2017). We also note that both A2261 and RXC0142.0+2131 show traces of the occurrence of minor mergers. However, the non-cool-core cluster A1576, which presents hints of a minor merger, does not host diffuse emission.

Many head-tail radio galaxies have been observed in the cluster environment, however a clear connection with the central diffuse emission is only seen in the case of RXJ1720.1+2638, where the tail might provide seed electrons for re-acceleration.

\section{Limits on cosmic-ray protons}

In this section, we aim to use some of our results to constrain the energy density of relativistic protons in the ICM by computing the maximum possible radio emission that can 


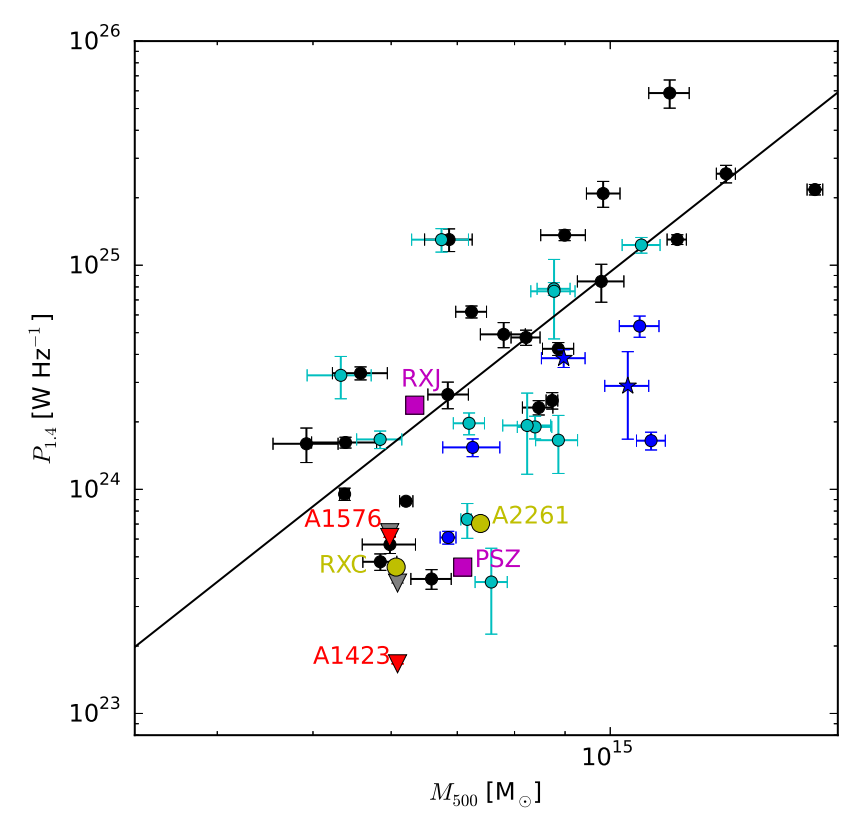

Fig. 14. Radio power at $1.4 \mathrm{GHz}$ vs. cluster mass $M_{500}$ for a sample of clusters with radio halo. The plot is reproduced from Martinez Aviles et al. (2016). Haloes with flux density measured at $1.4 \mathrm{GHz}$ are indicated by black circles and their related fit is shown as a black line. Haloes with flux density measured at frequencies other than $1.4 \mathrm{GHz}$ are indicated by cyan circles, ultra-steep haloes by blue circles, and ultra-steep haloes with flux density measured at frequencies other than $1.4 \mathrm{GHz}$ by blue stars. The upper limit of radio halo power at $1.4 \mathrm{GHz}$ in A1423 and A1576 are indicated by triangles, as derived in the literature in grey and the new limits obtained with LOFAR in red. The limit derived for A1576 is almost coincident with the value in literature, while that for A1423 is almost a factor of 2 smaller. We also indicate the power of the haloes in A2261 and RXCJ0142.0+2131 with yellow circles, and the steep-spectrum sources in PSZ1G139.6+24 and RXJ1720.1+2638 with magenta squares.

originate from hadronic collisions (Blasi \& Colafrancesco 1999; Dolag \& Enßlin 2000). We use a similar albeit more complicated procedure as in Brunetti et al. (2007), which we describe in the following.

The total admissible energy density of cosmic rays is given by requiring that this radio emission does not exceed our upper limit, given a model for the thermal gas pressure and the magnetic fields.

In this work, we place new upper limits on the radio power for the clusters A1576 and A1423. While the new upper limit for A1576 is comparable to the value previously derived in literature, for A1423 we were able to obtain a limit that is almost a factor of two deeper. Hence, we created a model for the gas density and temperature of A1423 following Ghirardini et al. (2019), who presented best-fit formulas for the thermodynamical properties of cool-core and non-cool-core clusters based on the analysis of a sample of clusters observed with XMM-Newton. We rescaled the values to the cluster mass and temperature ${ }^{7}$ of A1423, $M_{500}=$ $6.04 \times 10^{14} M_{\odot}$ and $T_{500}=6.4 \mathrm{keV}$.

We assumed that the magnetic field in A1423 is similar to the best-fit 3D model of the magnetic field in the Coma cluster presented in Bonafede et al. (2010) owing to the similarity in the cluster total mass. We generated a power-law distribution of the magnetic vector potential $A$ in Fourier space for a $(256)^{3}$ grid with

\footnotetext{
7 We used the core-excised temperature given by Giacintucci et al.
} (2017). a fixed resolution of $\Delta x=10 \mathrm{kpc}$ that is randomly drawn from the Rayleigh distribution. The magnetic field in real space follows $B=\nabla \times A$, which ensures that $\nabla \cdot B=0$ by construction. As in Coma, we assumed that the maximum coherence scale of the field is $\sim 40 \mathrm{kpc}$, the power law of fluctuations exhibits a Kolmogorov spectrum and the average magnetic field strength in the centre is $B_{0} \sim 4 \mu \mathrm{G}$. The volume-averaged magnetic field within a radius equal to $R_{\mathrm{H}}$ (i.e. the radius used for the upper limit, $436 \mathrm{kpc}$ for $\mathrm{A} 1423)$ is $\langle B\rangle=1.2 \mu \mathrm{G}$.

To be self-consistent with the profile of the mock halo that we injected, we assumed that the ratio of the spatial distribution of the cosmic-ray energy density, $E_{\mathrm{CR}}$, over the gas energy density profile, $E_{\text {gas }}$, is

$\frac{E_{\mathrm{CR}}}{E_{\mathrm{gas}}}=\left(\frac{E_{\mathrm{CR}}}{E_{\mathrm{gas}}}\right)_{0}\left(\frac{r}{\Delta x}\right)^{\alpha_{\mathrm{CR}}}$,

where the index 0 indicates the ratio computed at the cluster centre, and $\alpha_{\mathrm{CR}}$ is a shape parameter that allows for the non-linear scaling between cosmic rays and gas matter, as in Donnert et al. (2010) and Brunetti et al. (2012). We fixed $\alpha_{\mathrm{CR}}=1$ based on the requirement that the profile of the simulated radio emission within $1 \sigma$ (see below) matches the input halo model used in Sect. 4.5. We also imposed that the ratio $E_{C R} / E_{\text {gas }}$ is no larger than 0.1 .

The radio emission from hadronic collisions was derived following Pfrommer \& Enßlin (2004), i.e. assuming a Dermer model to compute the cross section of the protonproton interaction, and integrating the CRp population from $E_{\min }=0.1 \mathrm{GeV}$ to $E_{\max }=100 \mathrm{GeV}$ for a particle spectrum of $\alpha_{p}=2.6$, corresponding to a radio spectral index of $\alpha \sim-1.3$. Finally, we projected the emission and measured the flux density within a radius $R_{\mathrm{H}}$ from the cluster centre, and compared it to the upper limit we derived from the LOFAR observation of A1423.

Through this procedure, we find that $\left(\frac{E_{\mathrm{CR}}}{E_{\mathrm{gas}}}\right)_{0} \sim 0.1 \%$ yields $\left(\frac{E_{\mathrm{CR}}}{E_{\mathrm{gas}}}\right) \sim 3 \%$, averaged on the volume of a sphere of radius $R_{\mathrm{H}}$.

A comparison with the limit derived from the lack of hadronic $\gamma$-ray emission reported by the Fermi satellite for a sample of galaxy clusters (e.g. Ackermann et al. 2014) is not straightforward, and would require, for instance, a more detailed treatment of the cosmic-ray energy density distribution. However, we note that the limit we derived is of the same order of magnitude. Our constrain demonstrates the great potential of future, deeper LOFAR observations to constrain the energy budget of CRp in the ICM.

\section{Summary}

In this paper, we have presented the results of the largest campaign of LOFAR observations targeting galaxy clusters so far. The mass-selected sample consists of clusters with no sign of major mergers observed in the frequency range between $120 \mathrm{MHz}$ and $168 \mathrm{MHz}$. Data reduction was performed following the facet calibration scheme. Below we summarise our main results:

1. We find central, diffuse emission in the form of mini haloes surrounded by USS haloes in the cool-core clusters RXJ1720.1+2638 and PSZ1G139.6+24. Hence, we argue that the sloshing of the dense core after a minor merger can be responsible for the formation, not only of a central mini halo, but also of larger-scale emission that is visible at low radio frequencies. Moreover, the presence of a cool core, indicated by high values of the concentration parameter, $c$, and low values of the central entropy, $K_{0}$, might be significant for the 

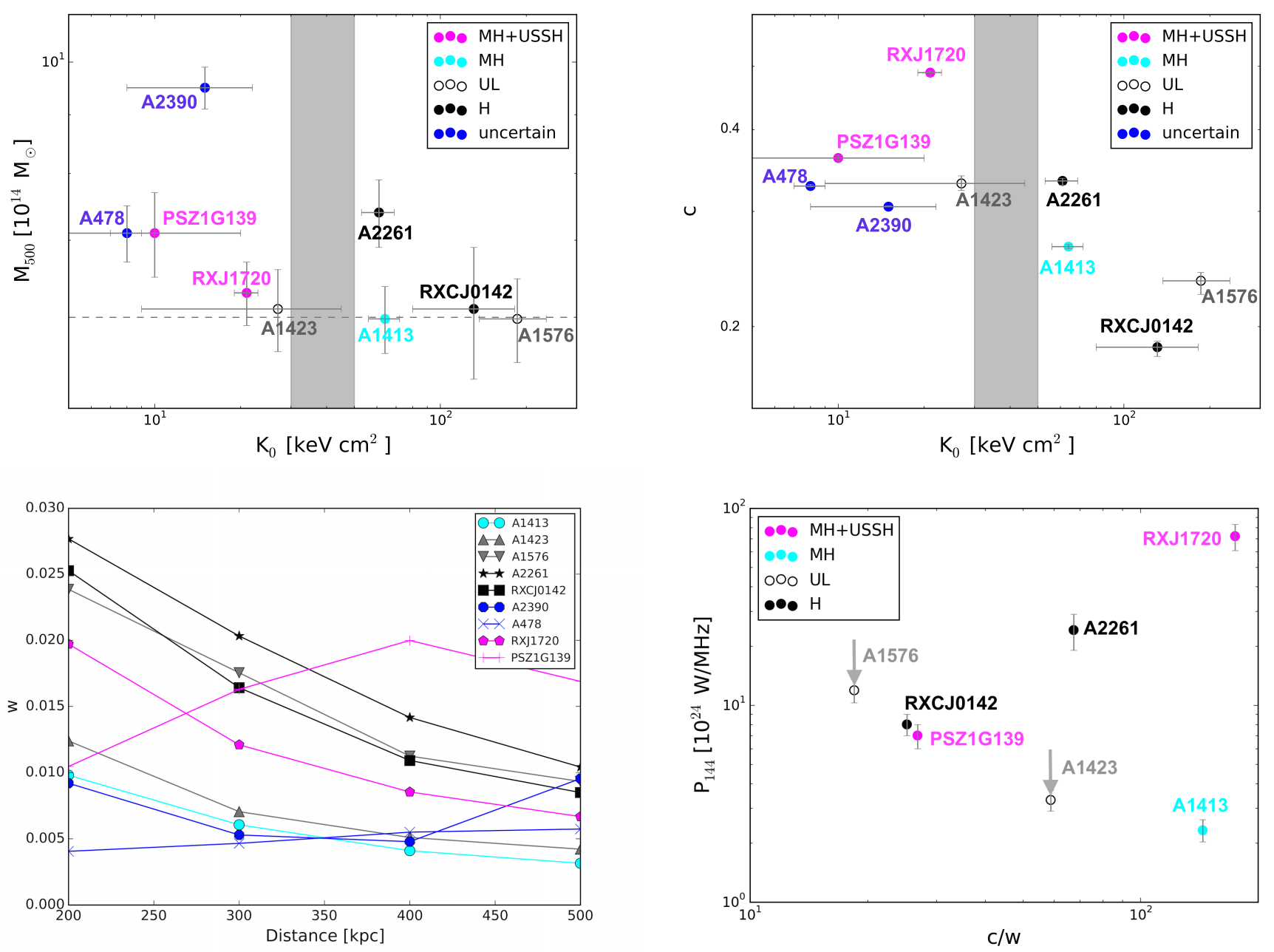

Fig. 15. Top left panel: central entropy $K_{0}$ vs. cluster mass $M_{500}$ of our selected sample of clusters. The selection in mass $\left(M_{500} \geq 6 \times 10^{14} M_{\odot}\right)$ is indicated by a dashed line. Clusters with low central entropies $\left.\left(K_{0}<30-50 \mathrm{keV} \mathrm{cm}\right)^{2}\right)$ are expected to host a cool core, whilst high central entropy $\left(K_{0}>50 \mathrm{keV} \mathrm{cm}{ }^{2}\right)$ indicates a non-cool-core cluster (Giacintucci et al. 2017). The sources are coloured according to the new LOFAR findings: clusters with mini haloes and USS haloes (MH+USSH) are indicated by magenta circles, with candidate mini haloes (cMH) by cyan circles and radio haloes $(\mathrm{H})$ by black circles. Clusters with no detected central diffuse radio emission, for which new upper limits (UL) were derived, are denoted by empty circles. Top right panel: central entropy $K_{0}$ vs. concentration parameter $c$. High values of $c$ and low values of $K_{0}$ indicate that the cluster host a cool core. In particular, clusters with low central entropies $\left(K_{0}<30-50 \mathrm{keV} \mathrm{cm}^{2}\right)$ host a cool core, whilst high central entropy $\left(K_{0}>50 \mathrm{keV} \mathrm{cm}^{2}\right)$ indicates a non-cool-core cluster (Giacintucci et al. 2017). The only warm-core cluster hosting a mini halo is A1413. Bottom left panel: emission centroid shift $w$ vs. distance from the cluster centre at which $w$ is computed. The value $w$ was computed at a scale of 200 , 300,400 and $500 \mathrm{kpc}$. We note that the value of $w$ gets smaller going to outer distances for all the clusters but PSZ1G139.61+24. Bottom right panel: total radio power at $144 \mathrm{MHz}$ of the diffuse emission vs. the ratio between the concentration parameter and emission centroid shift derived at $500 \mathrm{kpc}$.

formation of radio diffuse emission on scales larger than the cluster core that hosts a mini halo.

2. We confirm the presence of a mini halo in the non-cool-core cluster A1413 as proposed by Govoni et al. (2009).

3. We discover a radio halo in the non-cool-core cluster RXCJ0142.0+2131 with a scale of $570 \mathrm{kpc}$ and a spectral index of $\alpha_{144}^{610}<-1.3$.

4. We confirm the presence of a radio halo in the non-cool-core cluster A2261 as proposed by Sommer et al. (2017).

5. The central radio galaxy discovered in the massive cool-core cluster A2390 might account for most or even all the radio flux that was attributed to the giant radio halo proposed by Sommer et al. (2017); high-resolution observations in the frequency range between $144 \mathrm{GHz}$ and $1.4 \mathrm{GHz}$ are needed to confirm the morphology of the two radio jets and lobes and exclude the presence of the giant radio halo.
6. At LOFAR frequencies no centrally-located diffuse emission is observed in the cool-core cluster A478. We injected a mock mini halo, and placed a limit on the spectrum, i.e. $\alpha>-1$.

7. No cluster-scale diffuse radio emission is found in the cluster A1576 and in the cool-core cluster A1423 at the sensitivity of the observations, hence we derive new upper limits on the total radio power.

8. We use the limit on the radio power of A1423 to constrain the energy budget of CRp in the ICM and compare the result with the constraints derived from the lack of hadronic $\gamma$-ray emission reported by the Fermi satellite. We find that our LOFAR observations are competitive with the deepest limits derived by Fermi for the Coma cluster.

9. We discover head-tail radio galaxies in the clusters A1423 and A1413. We note also the presence of head-tail radio galaxies in A478, and in RXJ1720.1+2638, where the tail 
appears to be connected to the central source, and it might be a possible source of seed particles that can be re-accelerated. 10. No giant radio haloes in the cool-core clusters of our sample are found.

Low-frequency radio observations are ideal for discovering diffuse emission, in particular steep-spectrum emission from lowenergy CRe in galaxy clusters. Future studies performed on a larger sample of clusters will provide statistical information and help to further investigate the connection between the formation of radio emission from the ICM and its dynamical state.

Acknowledgements. This paper is based (in part) on data obtained with the International LOFAR Telescope (ILT) under the project codes listed in Table 2. LOFAR (van Haarlem et al. 2013) is the LOw Frequency ARray designed and constructed by ASTRON. It has observing, data processing, and data storage facilities in several countries, which are owned by various parties (each with their own funding sources) and are collectively operated by the ILT foundation under a joint scientific policy. The ILT resources have benefitted from the following recent major funding sources: CNRS-INSU, Observatoire de Paris and Université d'Orléans, France; BMBF, MIWF-NRW, MPG, Germany; Science Foundation Ireland (SFI), Department of Business, Enterprise and Innovation (DBEI), Ireland; NWO, The Netherlands; The Science and Technology Facilities Council, UK; Ministry of Science and Higher Education, Poland. Part of this work was carried out on the Dutch national e-infrastructure with the support of the SURF Cooperative through grant e-infra 160022 \& 160152. The LOFAR software and dedicated reduction packages on https://github.com/apmechev/GRID_LRT were deployed on the e-infrastructure by the LOFAR e-infragroup, consisting of J. B. R. Oonk (ASTRON and Leiden Observatory), A. P. Mechev (Leiden Observatory) and T. Shimwell (Leiden Observatory) with support from N. Danezi (SURFsara) and C. Schrijvers (SURFsara). This research made use of the NASA/IPAC Extragalactic Database (NED), which is operated by the Jet Propulsion Laboratory, California Institute of Technology, under contract with the National Aeronautics and Space Administration. A. Bonafede acknowledge support from the ERC-StG grant DRANOEL, n. 714245. F. Vazza acknowledges financial support from the ERC Starting Grant MAGCOW, no.714196. H. Rottgering and R. van Weeren acknowledge support from the ERC Advanced Investigator programme NewClusters 321271 and the VIDI research programme with project number 639.042 .729 , which is financed by the Netherlands Organisation for Scientific Research (NWO). F. de Gasperin is supported by the VEN research programme with project number 1808 , which is financed by the Netherlands Organisation for Scientific Research (NWO). A. Drabent acknowledges support by the BMBF Verbundforschung under the grant 05A17STA.

\section{References}

Ackermann, M., Ajello, M., Albert, A., et al. 2014, ApJ, 787, 18 Augusto, P., Edge, A. C., \& Chandler, C. J. 2006, MNRAS, 367, 366 Bacchi, M., Feretti, L., Giovannini, G., \& Govoni, F. 2003, A\&A, 400, 465 Barr, J., Jørgensen, I., Chiboucas, K., Davies, R., \& Bergmann, M. 2006, ApJ, 649, L1

Becker, R. H., White, R. L., \& Helfand, D. J. 1995, ApJ, 450, 559

Blasi, P., \& Colafrancesco, S. 1999, Astropart. Phys., 122, 169

Böhringer, H., Pratt, G. W., Arnaud, M., et al. 2010, A\&A, 514, A32

Bonafede, A., Feretti, L., Murgia, M., et al. 2010, A\&A, 513, A30

Bonafede, A., Intema, H. T., Brüggen, M., et al. 2014, MNRAS, 444, L44

Bonafede, A., Cassano, R., Brüggen, M., et al. 2017, MNRAS, 470, 3465

Botteon, A., Gastaldello, F., \& Brunetti, G. 2018, MNRAS, 476, 5591

Brienza, M., Morganti, R., Shulevski, A., Godfrey, L., \& Vilchez, N. 2016, Astron. Nachr., 337, 31

Briggs, D. S. 1995, PhD Thesis, New Mexico Institute of Mining Technology, Socorro, New Mexico, USA

Brunetti, G., \& Jones, T. W. 2014, Int. J. Mod. Phys. D, 23, 1430007

Brunetti, G., Venturi, T., Dallacasa, D., et al. 2007, ApJ, 670, L5

Brunetti, G., Giacintucci, S., Cassano, R., et al. 2008, Nature, 455, 944

Brunetti, G., Blasi, P., Reimer, O., et al. 2012, MNRAS, 426, 956
Buote, D. A. 2001, ApJ, 553, L15

Cassano, R., Brunetti, G., \& Setti, G. 2006, MNRAS, 369, 1577

Cassano, R., Brunetti, G., Setti, G., Govoni, F., \& Dolag, K. 2007, MNRAS, 378, 1565

Cassano, R., Brunetti, G., Venturi, T., et al. 2008, A\&A, 480, 687

Cassano, R., Ettori, S., Giacintucci, S., et al. 2010, ApJ, 721, L82

Cassano, R., Ettori, S., Brunetti, G., et al. 2013, ApJ, 777, 141

Cassano, R., Brunetti, G., Giocoli, C., \& Ettori, S. 2016, A\&A, 593, A81

Chandra, P., Ray, A., \& Bhatnagar, S. 2004, ApJ, 612, 974

Cuciti, V., Cassano, R., Brunetti, G., et al. 2015, A\&A, 580, A97

Dahle, H., Kaiser, N., Irgens, R. J., Lilje, P. B., \& Maddox, S. J. 2002, ApJS 139,313

de Gasperin, F., Intema, H. T., \& Shimwell, T. W. 2017, Sci. Adv., 3, e1701634

de Gasperin, F., Dijkema, T. J., Drabent, A. et al. 2019, A\&A, 622, A5 (LOFAR SI)

Dolag, K., \& Enßlin, T. A. 2000, A\&A, 362, 151

Donnert, J., Dolag, K., Brunetti, G., Cassano, R., \& Bonafede, A. 2010, MNRAS, 401, 47

Feretti, L., Giovannini, G., Govoni, F., \& Murgia, M. 2012, A\&ARv, 20, 54

Ghirardini, V., Eckert, D., Ettori, S., et al. 2019, A\&A, 621, A41

Giacintucci, S., Markevitch, M., Brunetti, G., et al. 2014a, ApJ, 795, 73

Giacintucci, S., Markevitch, M., Venturi, T., et al. 2014b, ApJ, 781, 9

Giacintucci, S., Markevitch, M., Cassano, R., et al. 2017, ApJ, 841, 71

Gitti, M., Brunetti, G., \& Setti, G. 2002, A\&A, 386, 456

Gitti, M., Ferrari, C., Domainko, W., Feretti, L., \& Schindler, S. 2007, A\&A, 470, L25

Govoni, F., Murgia, M., Markevitch, M., et al. 2009, A\&A, 499, 371

Haslam, C. G. T., Salter, C. J., Stoffel, H., \& Wilson, W. E. 1995, Astronomy Data Image Library

Intema, H. T., Jagannathan, P., Mooley, K. P., \& Frail, D. A. 2017, A\&A, 598, A78

Kale, R., Venturi, T., Giacintucci, S., et al. 2013, A\&A, 557, A99

Kale, R., Venturi, T., Giacintucci, S., et al. 2015, A\&A, 579, A92

Lovisari, L., Forman, W. R., Jones, C., et al. 2017, ApJ, 846, 51

Macario, G., Venturi, T., Brunetti, G., et al. 2010, A\&A, 517, A43

Markevitch, M., Vikhlinin, A., \& Forman, W. R. 2003, in Matter and Energy in Clusters of Galaxies, ed. S. Bowyer, ASP Conf. Ser., 301, 37

Martinez Aviles, G., Ferrari, C., Johnston-Hollitt, M., et al. 2016, A\&A, 595, A116

McMullin, J. P., Waters, B., Schiebel, D., Young, W., \& Golap, K. 2007, in Astronomical Data Analysis Software and Systems XVI, eds. R. A. Shaw, F. Hill, \& D. J. Bell, ASP Conf. Ser., 376, 127

Murgia, M., Govoni, F., Markevitch, M., et al. 2009, A\&A, 499, 679

Offringa, A. R., McKinley, B., Hurley-Walker, N., et al. 2014, MNRAS, 444, 606

Pfrommer, C., \& Enßlin, T. A. 2004, A\&A, 413, 17

Planck Collaboration XXIX. 2014, A\&A, 571, A29

Planck Collaboration XIII. 2016, A\&A, 594, A13

Santos, J. S., Rosati, P., Tozzi, P., et al. 2008, A\&A, 483, 35

Savini, F., Bonafede, A., \& Brueggen, M. 2018a, MNRAS, 478, 2234

Savini, F., Bonafede, A., Brüggen, M., et al. 2018b, MNRAS, 474, 5023

Scaife, A. M. M., \& Heald, G. H. 2012, MNRAS, 423, L30

Shimwell, T. W., Luckin, J., Brüggen, M., et al. 2016, MNRAS, 459, 277

Shimwell, T. W., Röttgering, H. J. A., Best, P. N., et al. 2017, A\&A, 598, A104

Shulevski, A., Morganti, R., Barthel, P. D., et al. 2015, A\&A, 579, A27

Sommer, M. W., Basu, K., Intema, H., et al. 2017, MNRAS, 466, 996

Sonkamble, S. S., Vagshette, N. D., Pawar, P. K., \& Patil, M. K. 2015, Ap\&SS, 359,21

Sun, M., Jones, C., Murray, S. S., et al. 2003, ApJ, 587, 619

van Haarlem, M. P., Wise, M. W., Gunst, A. W., et al. 2013, A\&A, 556, A2

van Weeren, R. J., Brunetti, G., Brüggen, M., et al. 2016a, ApJ, 818, 204

van Weeren, R. J., Williams, W. L., Hardcastle, M. J., et al. 2016b, ApJS, 223, 2

Venturi, T., Giacintucci, S., Dallacasa, D., et al. 2008, A\&A, 484, 327

Venturi, T., Rossetti, M., Brunetti, G., et al. 2017, A\&A, 603, A125

von der Linden, A., Mantz, A., Allen, S. W., et al. 2014, MNRAS, 443, 1973

Wilber, A., Brüggen, M., Bonafede, A., et al. 2018, MNRAS, 476, 3415

ZuHone, J. A., Markevitch, M., Brunetti, G., \& Giacintucci, S. 2013, ApJ, 762, 78 


\section{Appendix A: Additional figures}
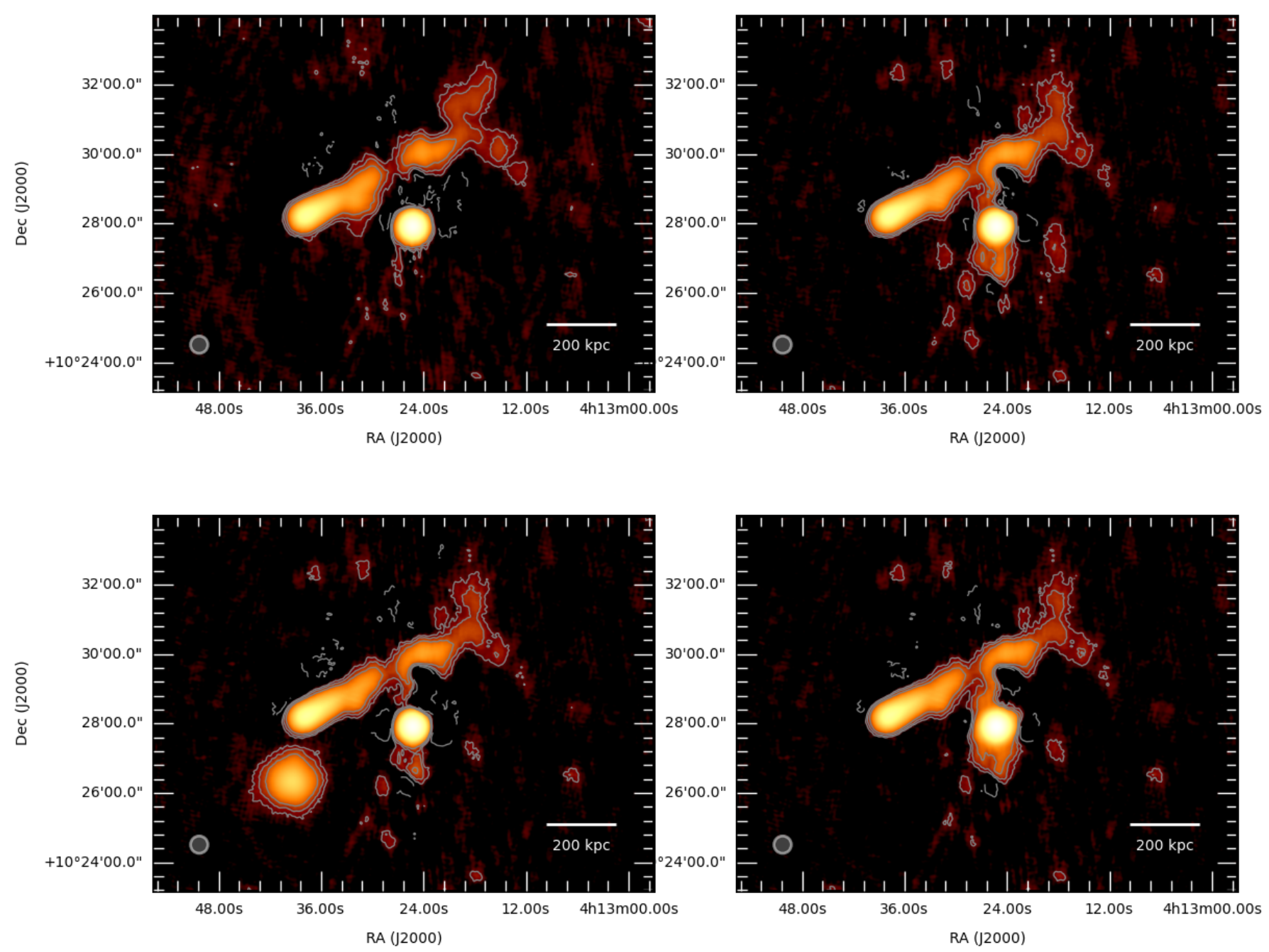

Fig. A.1. Low-resolution $\left(30^{\prime \prime} \times 30^{\prime \prime}\right)$ LOFAR image of the cluster A478 at $144 \mathrm{MHz}$. The contour levels are $(1,2,4) \times 3 \sigma$, where $\sigma=620 \mu \mathrm{Jy}_{\text {beam }}{ }^{-1}$. No mock mini halo is injected in the data set in the top left panel. A mock mini halo with $I_{0}=1 \mu \mathrm{Jy}$ arcsec ${ }^{-2}$ is injected at the cluster centre with $r_{\mathrm{e}}=100 \mathrm{kpc}$ in the top right panel. A mock mini halo with $I_{0}=13 \mu \mathrm{Jy} \mathrm{arcsec}{ }^{-2}$ is injected at the cluster centre with $r_{\mathrm{e}}=25 \mathrm{kpc}$ at the cluster centre in the bottom right panel, and in a close-by void region in the bottom left panel. The values for $r_{\mathrm{e}}$ and $I_{0}$ are chosen accordingly to the top panel of Fig. 7 in Murgia et al. (2009) and are referred to measurements at 1.4 GHz. 

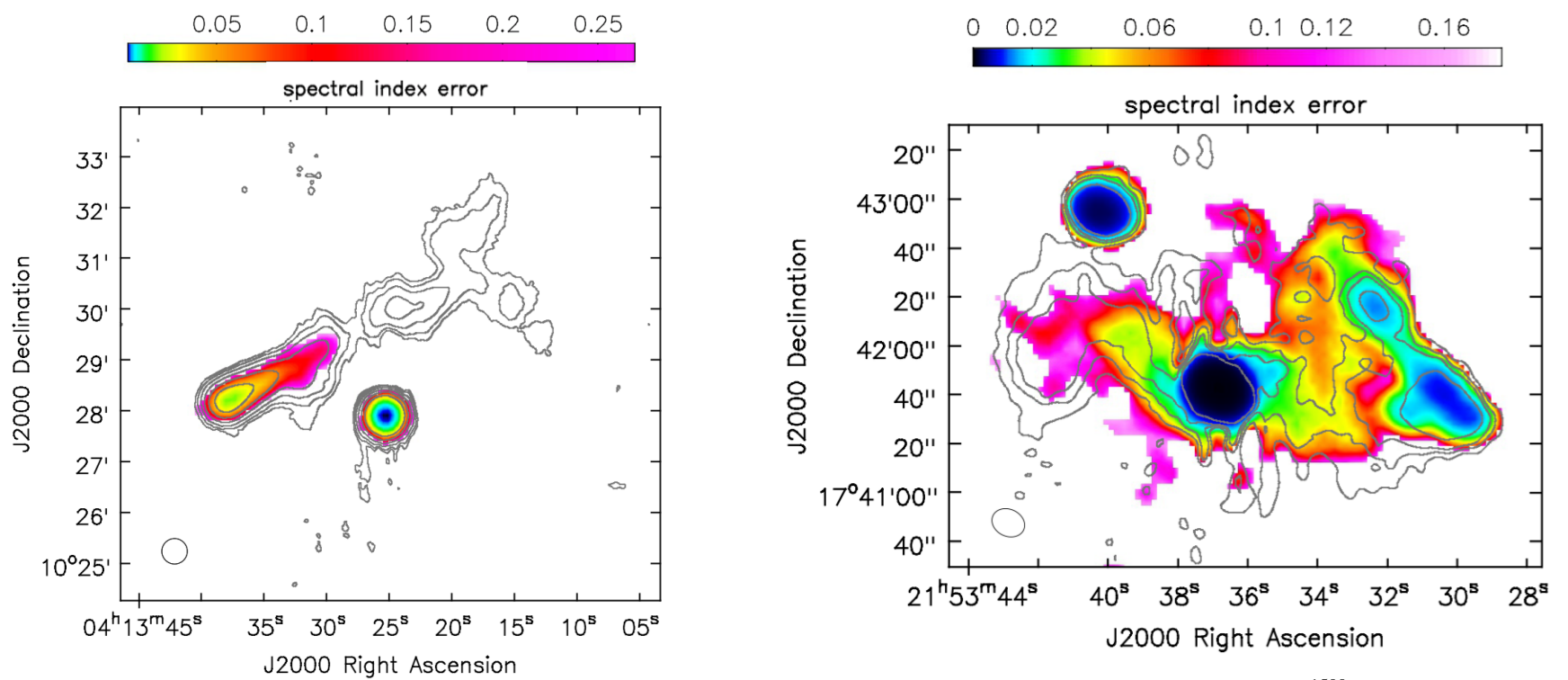

Fig. A.2. Spectral index error map of A478 $\left(\alpha_{144}^{610}\right)$ related to Fig. $4 . \quad \begin{aligned} & \text { Fig. A.4. Spectral index error } \\ & \text { Pixels below } 3 \sigma \text { are blanked. }\end{aligned}$ Pixels below $3 \sigma$ are blanked.

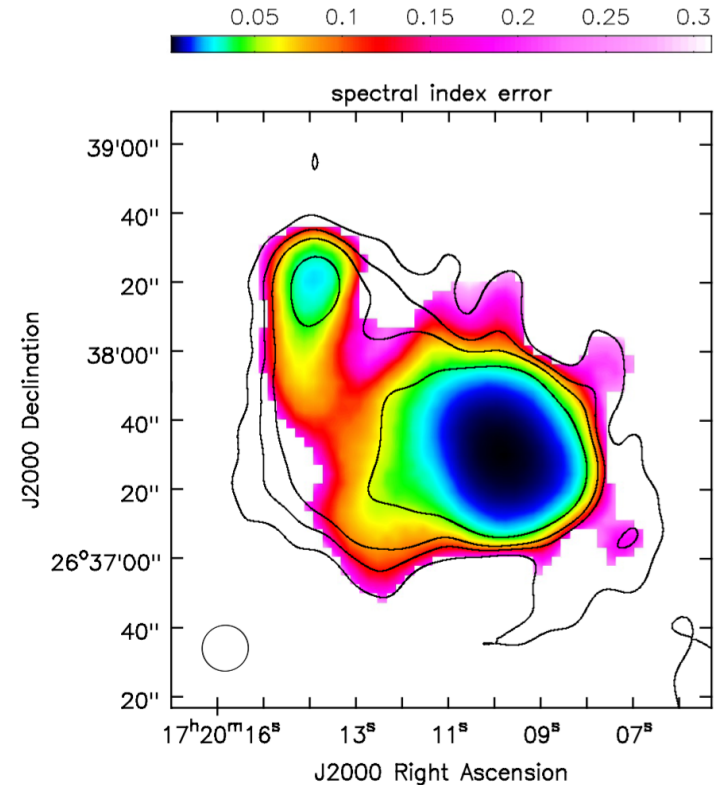

Fig. A.3. Spectral index error map of RXJ1720.1+2638 $\left(\alpha_{144}^{610}\right)$ related to Fig. 10. Pixels below $3 \sigma$ are blanked. 

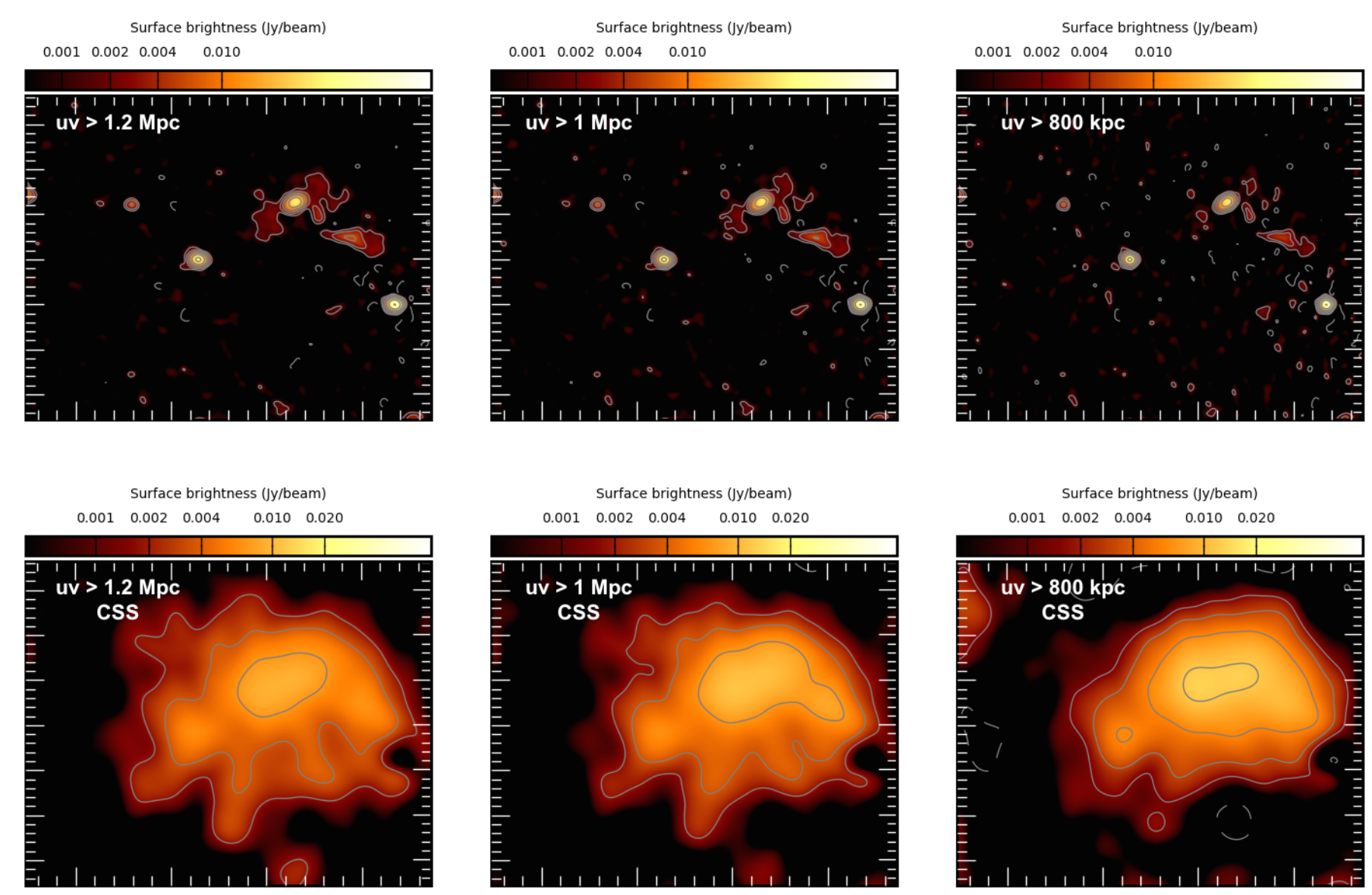

Fig. A.5. Top row: images obtained by changing the $u v$-range of the LOFAR observation of A2261 with different cuts. Contours start at $3 \sigma$, where $\sigma=450 \mu \mathrm{Jy} \mathrm{beam}^{-1}$, and are spaced by a factor of 2. Bottom row: corresponding $u v$-subtracted images. Different models of the discrete sources do not affect the detection of large-scale diffuse emission. Contours start at $3 \sigma$, where $\sigma=600 \mu \mathrm{Jy} \mathrm{beam}^{-1}$, and are spaced by a factor of 2 . 
A\&A 622, A24 (2019)

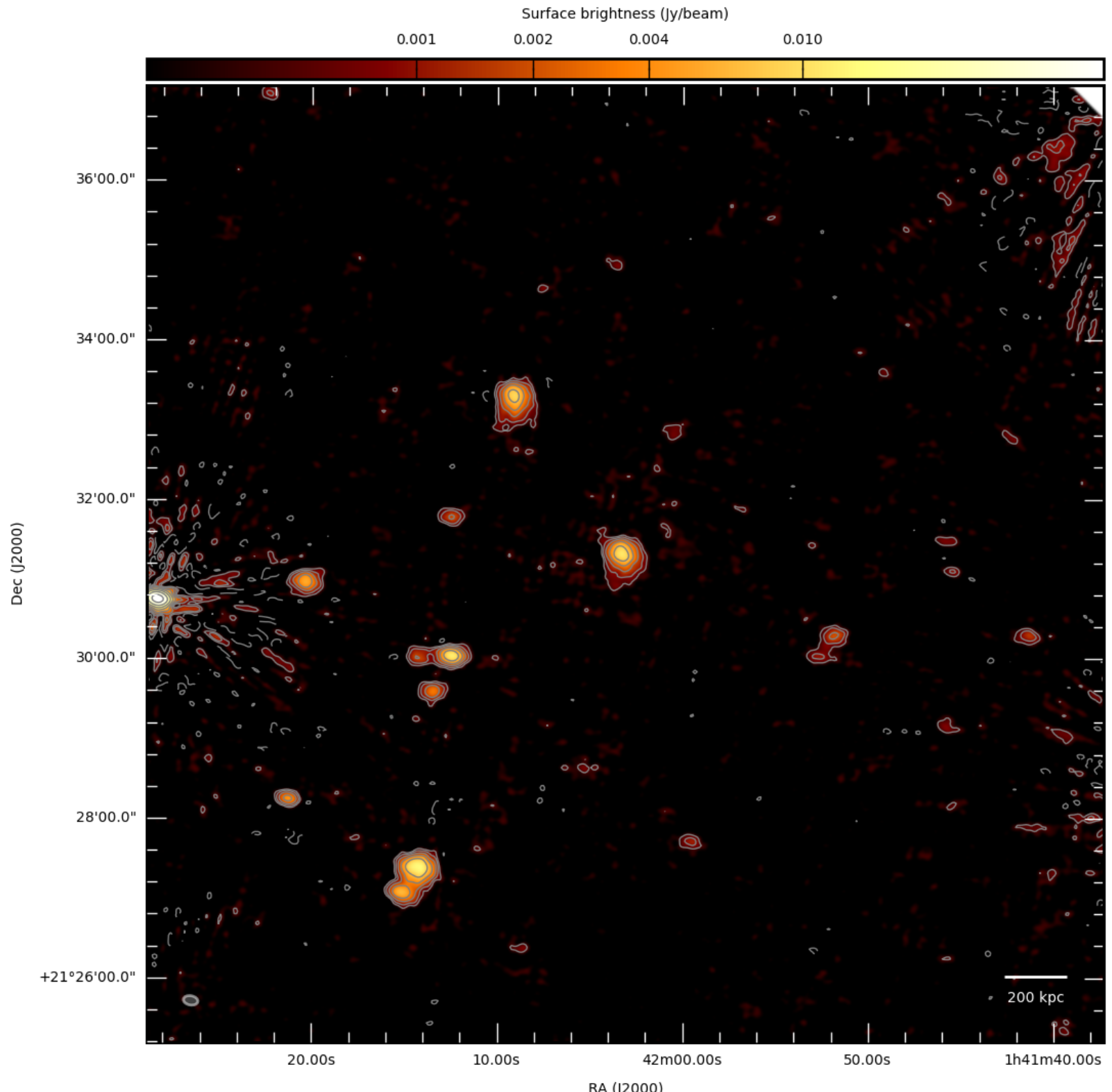

Fig. A.6. High-resolution $144 \mathrm{MHz}$ LOFAR image of RXCJ0142.0+2131. The contour levels start at $3 \sigma$, where $\sigma=150 \mu \mathrm{Jy}$ beam ${ }^{-1}$, and are spaced by a factor of two. The negative contour level at $-3 \sigma$ is overlaid with a dashed line. The beam is $11^{\prime \prime} \times 7^{\prime \prime}$ and is shown in grey in the bottom left corner of the image. 
F. Savini et al.: A LOFAR study of non-merging massive galaxy clusters

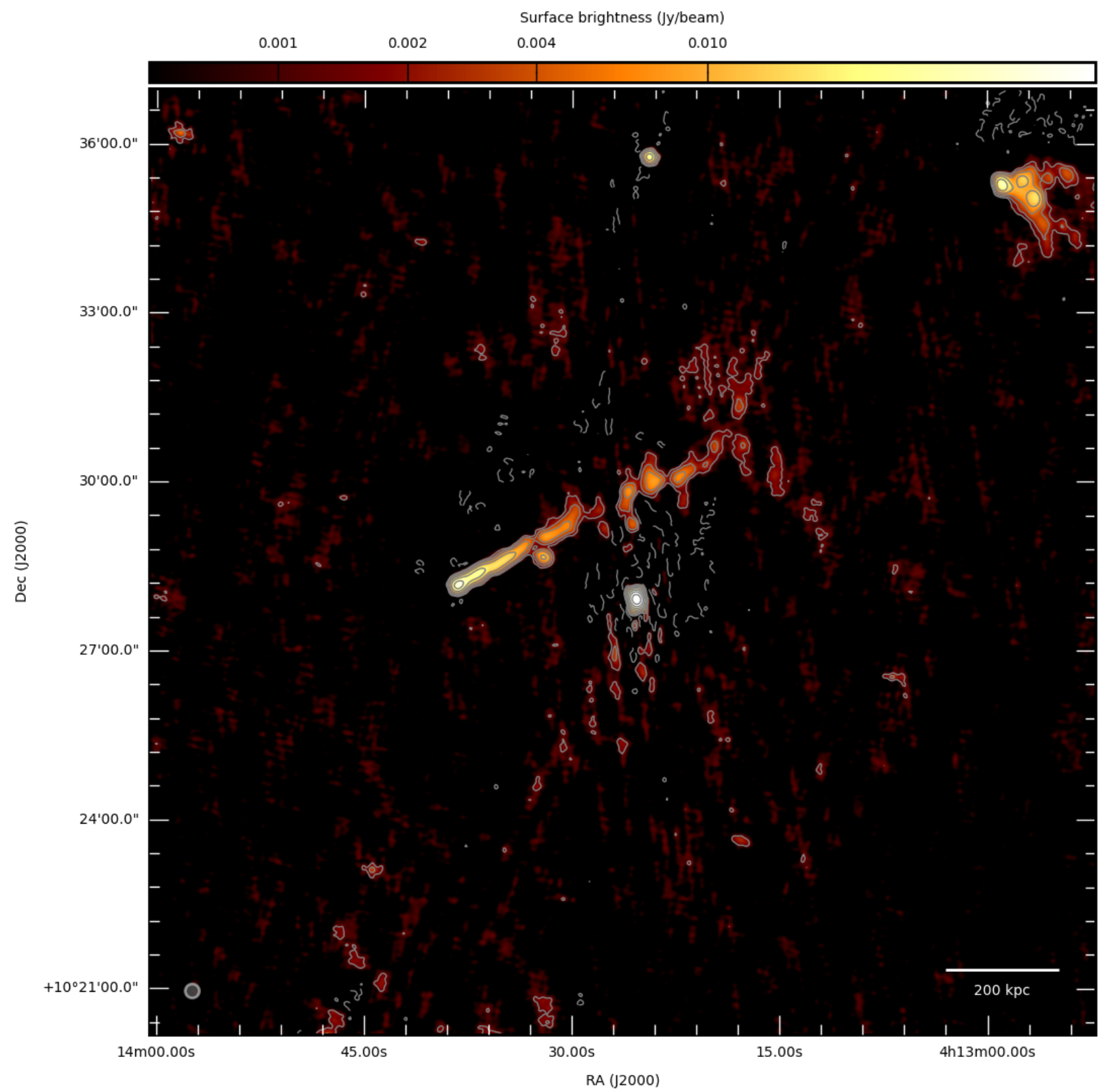

Fig. A.7. High-resolution $144 \mathrm{MHz}$ LOFAR image of A478. The contour levels start at $3 \sigma$, where $\sigma=450 \mu \mathrm{Jy}_{\text {beam }}{ }^{-1}$, and are spaced by a factor of two. The negative contour level at $-3 \sigma$ is overlaid with a dashed line. The beam is $15^{\prime \prime} \times 15^{\prime \prime}$ and is shown in grey in the bottom left corner of the image. We note the presence in the field of an head-tail radio galaxy located to the north-west of the cluster centre, which has an optical counterpart but no redshift information. 
A\&A 622, A24 (2019)

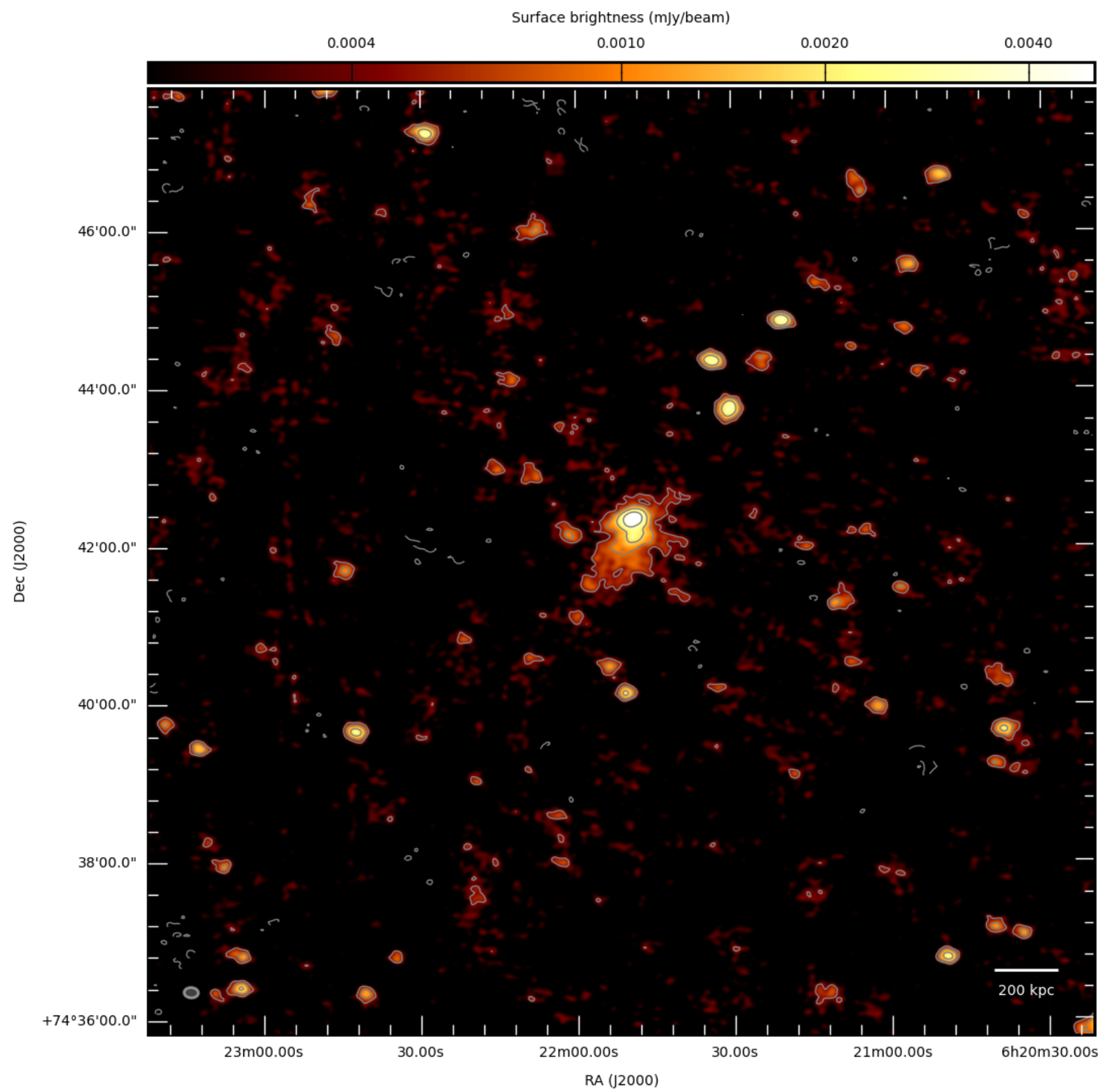

Fig. A.8. High-resolution $144 \mathrm{MHz}$ LOFAR image of PSZ1G139.61+24. The contour levels start at $3 \sigma$, where $\sigma=150 \mu \mathrm{Jy}$ beam ${ }^{-1}$, and are spaced by a factor of two. The negative contour level at $-3 \sigma$ is overlaid with a dashed line. The beam is $11^{\prime \prime} \times 8^{\prime \prime}$ and is shown in grey in the bottom left corner of the image. 
F. Savini et al.: A LOFAR study of non-merging massive galaxy clusters

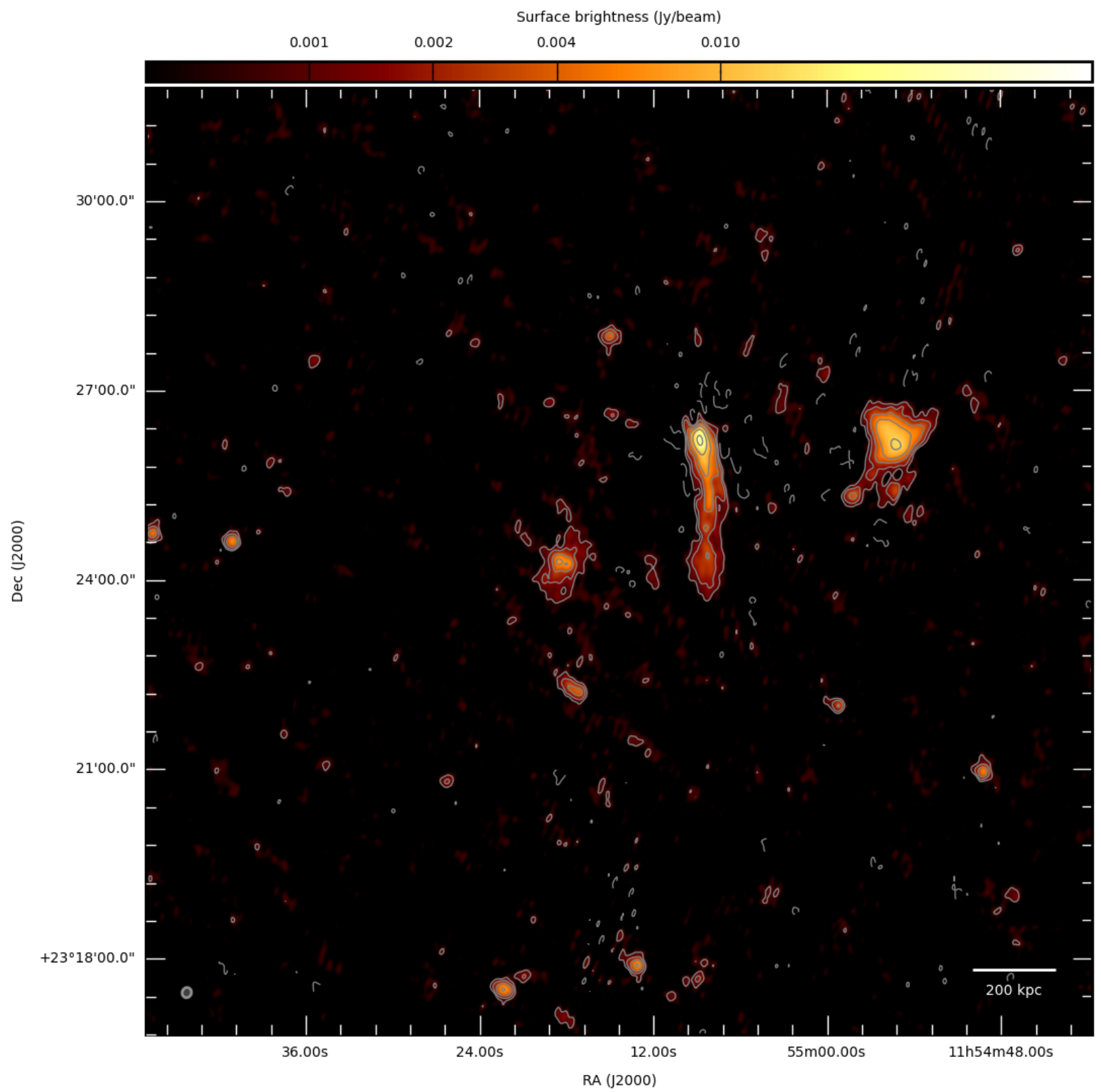

Fig. A.9. High-resolution $144 \mathrm{MHz}$ LOFAR image of A1413. The contour levels start at $3 \sigma$, where $\sigma=270 \mu \mathrm{Jy}_{\text {beam }}{ }^{-1}$, and are spaced by a factor of two. The negative contour level at $-3 \sigma$ is overlaid with a dashed line. The beam is $10^{\prime \prime} \times 9^{\prime \prime}$ and is shown in grey in the bottom left corner of the image. We note the presence in the field of a patch of diffuse emission located to the north-west of the cluster centre, which has no optical counter part. 
A\&A 622, A24 (2019)

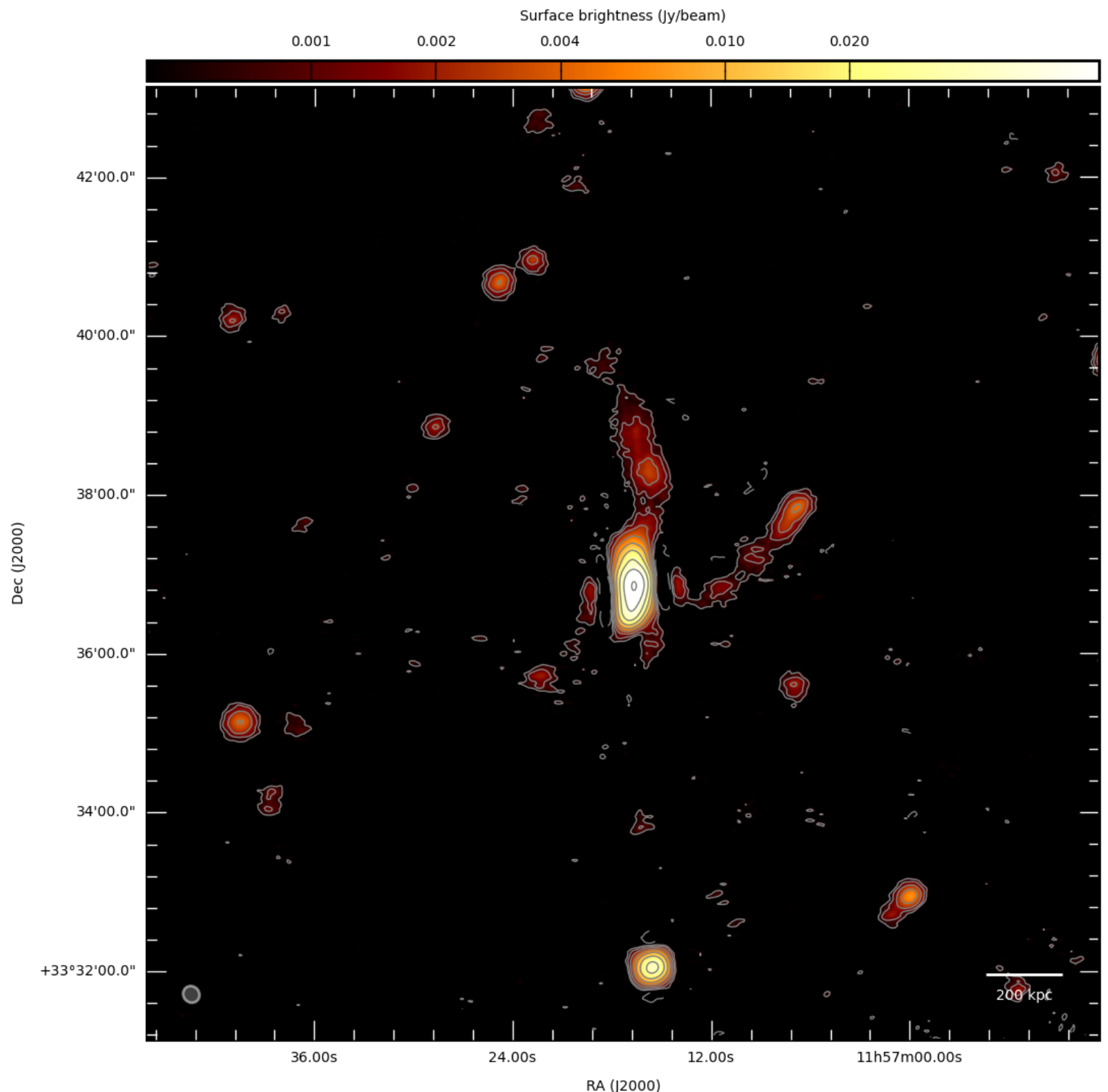

Fig. A.10. High-resolution $144 \mathrm{MHz}$ LOFAR image of A1423. The contour levels start at $3 \sigma$, where $\sigma=170 \mu \mathrm{Jy}$ beam ${ }^{-1}$, and are spaced by a factor of two. The negative contour level at $-3 \sigma$ is overlaid with a dashed line. The beam is $13^{\prime \prime} \times 12^{\prime \prime}$ and is shown in grey in the bottom left corner of the image. 
F. Savini et al.: A LOFAR study of non-merging massive galaxy clusters

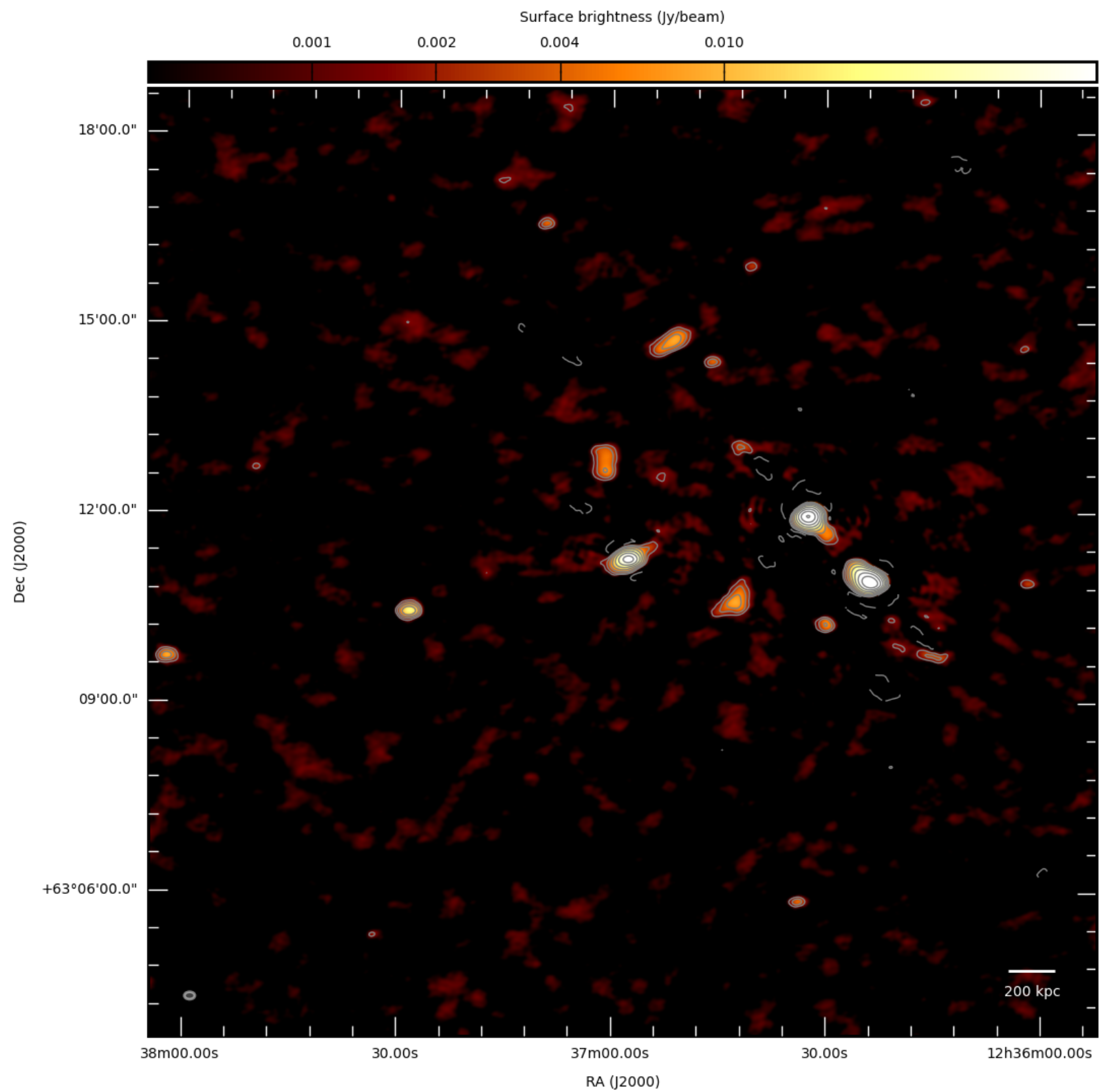

Fig. A.11. High-resolution $144 \mathrm{MHz}$ LOFAR image of A1576. The contour levels start at $3 \sigma$, where $\sigma=500 \mu \mathrm{Jy}_{\text {beam }}{ }^{-1}$, and are spaced by a factor of two. The negative contour level at $-3 \sigma$ is overlaid with a dashed line. The beam is $10^{\prime \prime} \times 7^{\prime \prime}$ and is shown in grey in the bottom left corner of the image. 
A\&A 622, A24 (2019)

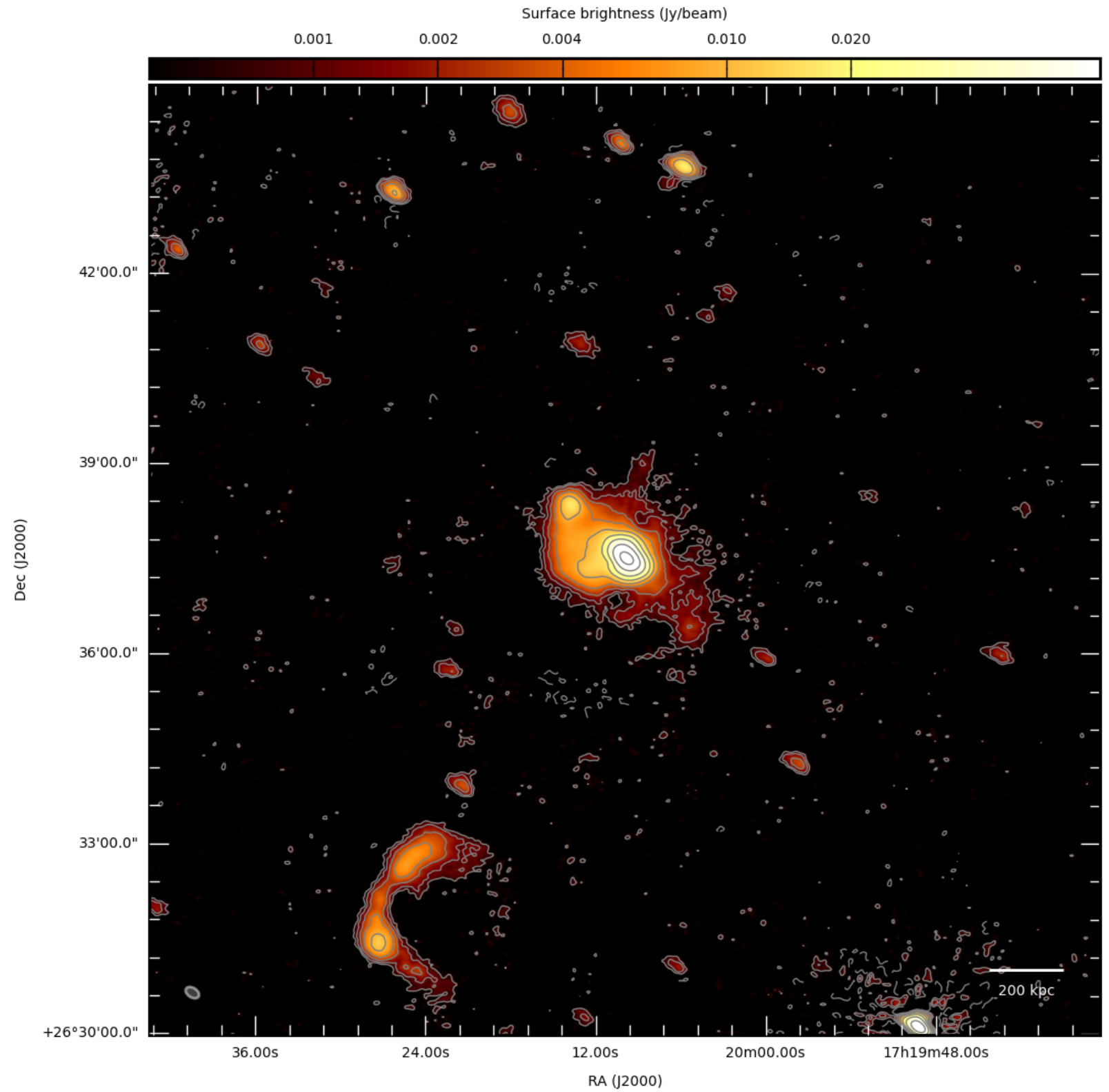

Fig. A.12. High-resolution $144 \mathrm{MHz}$ LOFAR image of RXJ1720.1+2638. The contour levels start at $3 \sigma$, where $\sigma=200 \mu \mathrm{Jy}_{\mathrm{beam}}{ }^{-1}$, and are spaced by a factor of two. The negative contour level at $-3 \sigma$ is overlaid with a dashed line. The beam is $14^{\prime \prime} \times 9^{\prime \prime}$ and is shown in grey in the bottom left corner of the image. We note the presence in the field of a wide-angle tail radio galaxy located to the south-east of the cluster centre, which is associated with a cluster member galaxy at $z=0.159$. 
F. Savini et al.: A LOFAR study of non-merging massive galaxy clusters

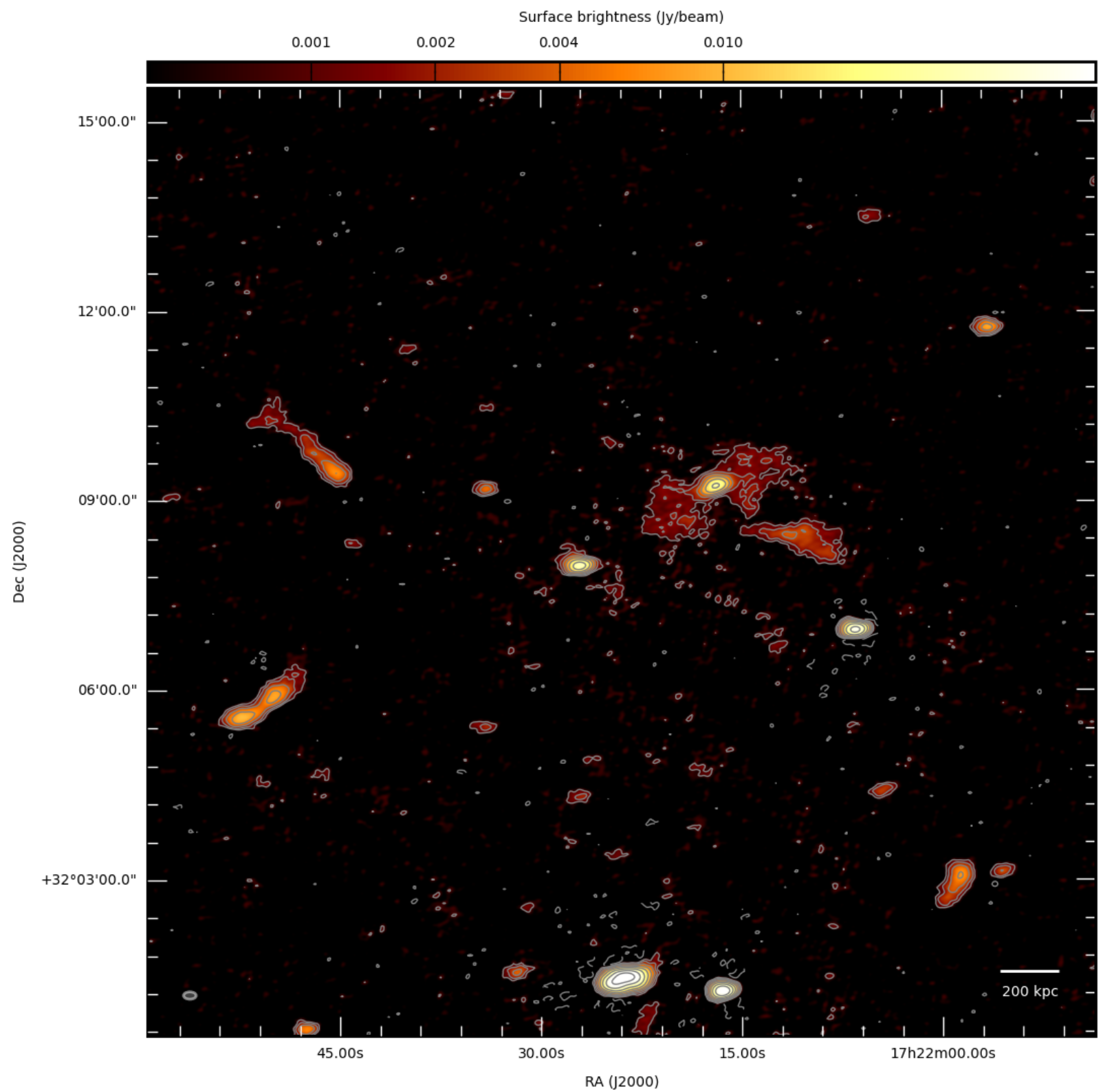

Fig. A.13. High-resolution $144 \mathrm{MHz}$ LOFAR image of A2261. The contour levels start at $3 \sigma$, where $\sigma=270 \mu \mathrm{Jy}$ beam $^{-1}$, and are spaced by a factor of two. The negative contour level at $-3 \sigma$ is overlaid with a dashed line. The beam is $12^{\prime \prime} \times 7^{\prime \prime}$ and is shown in grey in the bottom left corner of the image. We note the presence in the field of a tail located to the north-east of the cluster centre, which has an optical counterpart but no redshift information. 
A\&A 622, A24 (2019)

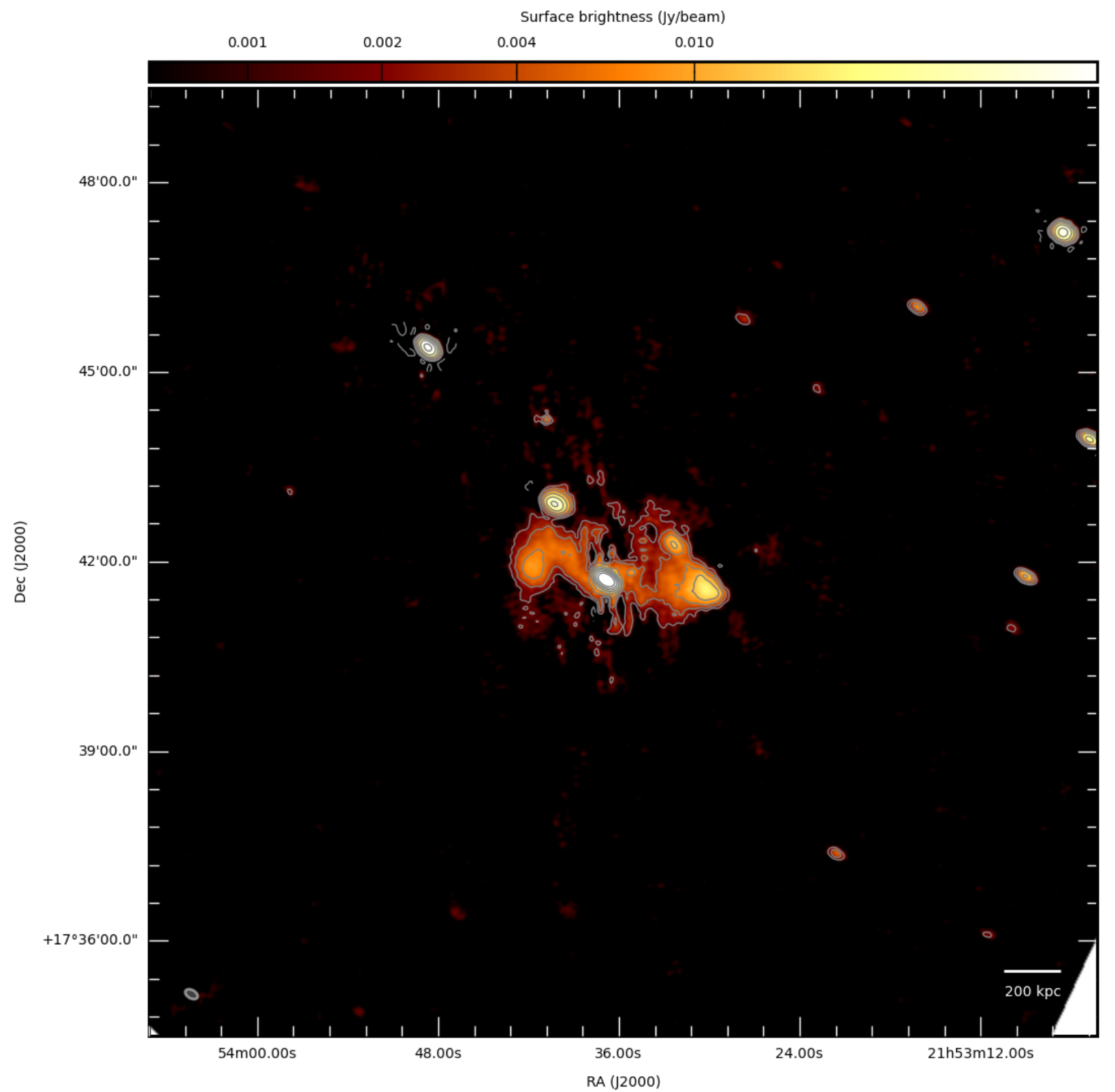

Fig. A.14. High-resolution $144 \mathrm{MHz}$ LOFAR image of A2390. The contour levels start at $3 \sigma$, where $\sigma=400 \mu \mathrm{Jy}_{\text {beam }}{ }^{-1}$, and are spaced by a factor of two. The negative contour level at $-3 \sigma$ is overlaid with a dashed line. The beam is $13^{\prime \prime} \times 8^{\prime \prime}$ and is shown in grey in the bottom left corner of the image. 